\title{
WEAK CONVERGENCE RESULTS FOR THE KAKUTANI INTERVAL SPLITTING PROCEDURE
}

\author{
By RONALD PYKE AND WILlEM R. VAN ZWET \\ University of Washington and University of Leiden
}

\begin{abstract}
This paper obtains the weak convergence of the empirical processes of both the division points and the spacings that result from the Kakutani interval splitting model. In both cases, the limit processes are Gaussian. For the division points themselves, the empirical processes converge to a Brownian bridge as they do for the usual uniform splitting model, but with the striking difference that its standard deviations are about one-half as large. This result gives a clear measure of the degree of greater uniformity produced by the Kakutani model. The limit of the empirical process of the normalized spacings is more complex, but its covariance function is explicitly determined. The method of attack for both problems is to obtain first the analogous results for more tractable continuous parameter processes that are related through random time changes. A key tool in their analysis is an approximate Poissonian characterization that obtains for cumulants of a family of random variables that satisfy a specific functional equation central to the $K$-model.
\end{abstract}

1. Introduction. We are interested in comparisons between two probability models for the random subdivision of the unit interval. The first is the usual model in which the division points are independent $\operatorname{Unif}(0,1)$ random variables (r.v.'s). We refer to this as the $U$-model. The second model will be referred to as the $K$-model (for Kakutani) in which the first division point, $X_{1}$, is a $\operatorname{Unif}(0,1)$ r.v., and then thereafter the $n$th division point, $X_{n}$, conditionally given the preceding $n-1$ points $\left\{X_{1}, \ldots, X_{n-1}\right\}$, is uniformly distributed over the largest subinterval formed by $0,1, X_{1}, X_{2}, \ldots, X_{n-1}$. The $K$-model was suggested by Kakutani (1975) who conjectured that the empirical distribution function (d.f.) of the first $n$ subdivision points converges to the uniform d.f. on $[0,1]$, just as is well known to be the case for the $U$-model. This Glivenko-Cantelli result for the $K$-model was shown to be true by van Zwet (1978).

The $K$-method of interval splitting, however, should by its very nature result in "more uniform" spacings than those of the $U$-method. This is intuitively clear since in the $K$-model the largest spacing is always the one that is being split, whereas in the $U$-model, the largest spacing may remain untouched for several iterations while at the same time the smaller intervals are consequently being divided into even smaller ones. This difference between the two models was clarified in Pyke (1980)

Received December 2001; revised December 2002.

AMS 2000 subject classifications. Primary 60F17; secondary 60G99, $62 \mathrm{G} 30$.

Key words and phrases. Empirical processes, Kakutani interval splitting, spacings, weak convergence, cumulants, self-similarity. 
where it was shown that for the $K$-model the empirical d.f. of the normalized spacings converges uniformly with probability one to the uniform d.f. on $[0,2]$. This is in sharp contrast to the $U$-model where the limit is an exponential d.f. over $(0, \infty)$; a result of Blum [cf. the footnote in Weiss (1955)].

The purpose of this paper is to study the weak convergence under the Kakutani model of the empirical processes for both the division points and their spacings. The results and their proofs clarify further the differences between the $U$ - and the $K$-model. The differences are rather striking. In particular, the difference between the two interval-splitting models is summarized by the fact that although the empirical processes for the division points converge in law to Brownian bridges under both the $U$ - and $K$-models, the standard deviations in the latter case are approximately half what they are for the former; see Theorem 4.1.

To be more precise we introduce the following notation. Let $\left\{X_{n}: n \geq 1\right\}$ be the sequence of r.v.'s with values in $(0,1)$ that represent the successive division points of the unit interval. Let $X_{n 1} \leq X_{n 2} \leq \cdots \leq X_{n n}$ be the ordered values of $\left\{X_{1}, \ldots, X_{n}\right\}$. Define the spacings

$$
D_{n i}=X_{n i}-X_{n, i-1}, \quad 1 \leq i \leq n+1 \text { with } X_{n 0}=0, X_{n, n+1}=1,
$$

and let $D_{n i}^{*}:=(n+1) D_{n i}, 1 \leq i \leq n+1$, denote the normalized spacings. Since under the $K$-model, the maximum normalized spacing converges a.s. to 2 [see (1.12)], it is expedient to introduce the relative spacings, $\left\{D_{n i} / M_{n} ; 1 \leq i \leq\right.$ $n+1\}$ in which $M_{n}:=\max \left\{D_{n 1}, \ldots, D_{n n+1}\right\}$.

Let $F_{n}, G_{n}$ and $G_{n}^{*}$ denote, respectively, the empirical d.f.'s of the division points $\left\{X_{1}, \ldots, X_{n}\right\}$, the spacings $\left\{D_{n 1}, \ldots, D_{n, n+1}\right\}$ and the normalized spacings $\left\{D_{n 1}^{*}, \ldots, D_{n, n+1}^{*}\right\}$. Let $F$ be the $\operatorname{Unif}(0,1)$ d.f., $G$ be the $\operatorname{Unif}(0,2)$ d.f., and $H$ be the exponential d.f. with mean 1. Then the Glivenko-Cantelli results reviewed above can be stated as follows, where $\|\cdot\|$ is the supremum norm in $\mathbb{R}^{1}$ : with probability 1 under the $U$-model,

$$
\left\|F_{n}-F\right\| \rightarrow 0 \text { and }\left\|G_{n}^{*}-H\right\| \rightarrow 0
$$

whereas under the $K$-model

$$
\left\|F_{n}-F\right\| \rightarrow 0 \text { and }\left\|G_{n}^{*}-G\right\| \rightarrow 0 .
$$

Thus no differentiation between the two models shows up at this level for the division points, though it does for the spacings. However, Theorem 4.1 shows dramatically that differences are in fact present for the division points in the orders of $n^{1 / 2}\left\|F_{n}-F\right\|$.

Before introducing the notation for the processes to be studied, we recall that the key method of proofs for results under the $K$-model involves a random time change from the discrete index $n \in \mathbb{Z}^{+}$to the continuous parameter $s>0$ defined by

$$
N_{s}=\min \left\{n \in \mathbb{Z}^{+}: M_{n} \leq s\right\}, \quad s>0,
$$


where $M_{0}=1$. Interpret $\min \varnothing=+\infty$. Note that $N_{s}=0$ when $s \geq 1$. Thus $N_{s}$ denotes the smallest sample size $n$ for which no spacing exceeds $s$. The method relies essentially upon a stochastic recursion relationship [(1.9) or (1.10)] that holds in the continuously indexed case. Results are first proved for this case and then an argument is provided to show that the results desired for the original quantities (indexed by $n$ ) follow as corollaries.

In terms of the parameter $s$, the analogous functions to those introduced above are

$$
\begin{aligned}
F(x, s) & =F_{N_{s}}(x), \\
N_{s}(x) & =N_{s} F(x, s)={ }^{\#}\left\{j: X_{j} \leq x, 1 \leq j \leq N_{s}\right\}, \\
G(x, s) & =G_{N_{s}}(x), \\
K(x, s) & =\left(N_{s}+1\right) G(x, s)={ }^{\#}\left\{j: D_{N_{s} j} \leq x, 1 \leq j \leq N_{s}+1\right\} .
\end{aligned}
$$

The following results from van Zwet (1978) and Pyke (1980) are used extensively throughout the paper:

$$
\begin{aligned}
\mu(t) & :=E N_{t}= \begin{cases}2 / t-1, & \text { for } 0<t<1, \\
0, & \text { for } t \geq 1,\end{cases} \\
v(t) & :=\operatorname{var} N_{t}=c / t \quad \text { for } 0<t \leq 1 / 2, \\
\mu(x, s) & :=E K(x, s)= \begin{cases}2 x / s^{2}, & \text { if } 0<x \leq s<1, \\
2 / s, & \text { if } 0<s<x \leq 1, \\
\varepsilon(x-1), & \text { if } s \geq 1,\end{cases}
\end{aligned}
$$

where $\varepsilon(u)=0$ or 1 according as $u<0$ or $u \geq 0$. The constant $c=v(1 / 2) / 2$ in (1.7) is evaluated in Lemma 3.2. A key result in this paper is Theorem 2.2 that shows in particular that all of the remaining cumulants of $N_{t}$ are also proportional to $t^{-1}$ in intervals of the form $(0,1 / k)$. Central to the study of these and all other results about the continuous parameter version of the Kakutani method are the recursive representations that come directly from the iterative nature of the Kakutani procedure. In particular, one may check that $N_{t}$ satisfies the relationship

$$
N_{t} \stackrel{L}{=} N_{t / U}+N_{t /(1-U)}^{*}+1, \quad 0<t<1,
$$

where $N$ and $N^{*}$ are independent identically distributed processes and $U$ is a Unif $(0,1)$ r.v. independent of $N$ and $N^{*}$. More generally, one can show that

$$
K(x, t) \stackrel{L}{=} K\left(\frac{x}{U}, \frac{t}{U}\right)+K^{*}\left(\frac{x}{1-U}, \frac{t}{1-U}\right), \quad 0<t<1,
$$

where again $K \stackrel{L}{=} K^{*}$ and $K, K^{*}$ and $U$ are independent. Of course, $U$ represents the first (uniform) partition point of the unit interval. Since $K(1, t)=K(t, t)=$ $N_{t}+1,(1.9)$ is seen to be a special case of (1.10). 
Throughout the paper we also need the following limit results from van Zwet (1978) and Pyke (1980) which are contained in their proofs of the GlivenkoCantelli results of (1.3):

$$
\begin{aligned}
& s N_{s} \rightarrow 2 \quad \text { a.s. as } s \rightarrow 0 \text {; } \\
& n M_{n} \rightarrow 2 \quad \text { a.s. as } n \rightarrow \infty \\
& \text { where } M_{n}=\max \left\{D_{n i}: 1 \leq i \leq n+1\right\} \text {; } \\
& s^{-1} M_{N_{s}} \rightarrow 1 \quad \text { a.s. as } s \rightarrow 0 \text { [from (1.11) and (1.12)]; } \\
& \text { (1.14) } \quad s K(y s, s) \rightarrow 2 y \quad \text { uniformly for } 0 \leq y \leq 1 \text {, a.s. as } s \rightarrow 0 \text {. }
\end{aligned}
$$

The purpose of this paper is to study under the $K$-model the weak convergence of the empirical processes associated with the division points and the spacings. We denote these processes of interest as follows:

(i) empirical processes of the division points: for $0 \leq x \leq 1$,

$$
\begin{array}{ll}
\text { parameter } n \geq 1, & U_{n}(x)=n^{1 / 2}\left\{F_{n}(x)-x\right\}, \\
\text { parameter } s>0, & U(x, s)=(s / 2)^{1 / 2}\left\{N_{s}(x)-x N_{s}\right\} ;
\end{array}
$$

(ii) empirical processes of the normalized spacings: for $0 \leq y \leq 1$,

$$
\begin{array}{ll}
\text { parameter } n \geq 1, & V_{n}^{*}(y)=n^{1 / 2}\left\{G_{n}^{*}(2 y)-y\right\}, \\
\text { parameter } s>0, & V^{*}(y, s)=(s / 2)^{1 / 2}\left\{K\left(\frac{2 y}{N_{s}+1}, s\right)-y\left(N_{s}+1\right)\right\} ;
\end{array}
$$

(iii) empirical processes of the relative spacings: for $0 \leq y \leq 1$,

$$
\begin{array}{ll}
\text { parameter } n \geq 1, & V_{n}(y)=\sqrt{n+1}\left\{G_{n}\left(M_{n} y\right)-y\right\}, \\
\text { parameter } s>0, & V(y, s)=(2 / s)^{1 / 2}\left\{\frac{K(y s, s)}{N_{s}+1}-y\right\} .
\end{array}
$$

For convenience, we will refer to processes indexed by continuous parameters as stopped processes, referring thereby to the random stopping times $N_{s}$ involved in their definitions.

Central to the study of these processes is the related stopped process defined by

$$
W(y, s)=(2 / s)^{1 / 2}\left\{\frac{s}{2} K(y s, s)-y\right\}, \quad 0 \leq y<\infty, s>0,
$$

since as we now show, $V$ and $V^{*}$ are expressible in terms of $W$ and $W$ is simpler to study. Observe that in view of $(1.8), W(\cdot, s)$ is a centered process only for $0 \leq y \leq 1$ and $s<1$. Since $N_{s}+1=K(s, s)$,

$$
W(1, s)=(2 / s)^{1 / 2}\left\{\frac{s}{2}\left(N_{s}+1\right)-1\right\}
$$


and one can check that with $\delta_{s}:=2 /\left(s\left(N_{s}+1\right)\right)$, the two stopped spacings processes satisfy

$$
V^{*}(y, s)=W\left(y \delta_{s}, s\right)-W(1, s)\left(\delta_{s}+1\right) y
$$

and

$$
\begin{aligned}
V(y, s) & =(2 / s)^{1 / 2}\left\{\frac{K(y s, s)}{N_{s}+1}-y\right\} \\
& =\frac{(2 / s)^{1 / 2}}{N_{s}+1}\left\{K(y s, s)-y\left(N_{s}+1\right)\right\} \\
& =\delta_{s}(2 / s)^{1 / 2}\left\{\frac{s}{2} K(y s, s)-y-\frac{s}{2} y\left(K(s, s)-\frac{2}{s}\right)\right\} \\
& =\delta_{s}\{W(y, s)-y W(1, s)\}
\end{aligned}
$$

for $0<s \leq 1$. Since $\delta_{s} \rightarrow 1$ a.s., by (1.11), the limiting behaviors of $V^{*}(\cdot, s)$ over $0<y<1$ and $V(\cdot, s)$ over $0<y \leq 1$ will follow from that of $W(\cdot, s)$ in $D[0,1]$. Notice that although $V^{*}$ may appear to be a type of "tied-down" version of $W$, it is not actually zero at $y=1$, as is $V$. Moreover, the support interval of significance for $V^{*}(\cdot, s)$ is random, namely, $\left[0, \delta_{s}\right]$. This is a result of the fact that the normalized maximum spacing has a finite limit; see (1.12). Since the limiting distribution of the maximum spacing may be obtained separately [see (6.6) and the discussion following] it suffices to place our emphasis here upon the processes of the relative spacings, namely, $V_{n}$ and $V(\cdot, s)$, which we do in Section 6.

The limiting behaviors of the empirical processes $U_{n}$ and $V_{n}^{*}$ under the $U$-model are well known. Essentially due to Donsker [(1952); cf. Billingsley (1968)] is the fact that $U_{n} \rightarrow_{L} U$, where $U$ is the standard Brownian bridge with representation $U(t)=B_{0}(t):=B(t)-t B(1), 0 \leq t \leq 1$, in which $B$ is the standard Brownian motion with $B(0)=0$ and $\operatorname{var} B(1)=1$. For the spacings' empirical process, weak convergence was obtained in Pyke [(1965), Theorem 6.4]. Here the definition must be modified to $V_{n}^{*}(y)=n^{1 / 2}\left\{G_{n}^{*}\left(H^{-1}(y)\right)-y\right\}$ to keep the process on $[0,1]$ since by (1.2) and (1.3) the a.s. limit for $G_{n}^{*}$ is the exponential $H$ rather than the uniform $G$ over $(0,2)$. Hence $H^{-1}(y)=-\ln (1-y)$. [In (1.16) observe that $G^{-1}(y)=2 y$.] With this notational change, the $U$-model's weak convergence result for the spacings' empirical process is that $V_{n}^{*} \rightarrow_{L} V^{*}$ where $V^{*}$ is a mean zero, Gaussian process with

$$
\operatorname{Cov}\left\{V^{*}(x), V^{*}(y)\right\}=x(1-y)-m(x) m(y), \quad 0 \leq x \leq y \leq 1,
$$

where $m(y)=-(1-y) \ln (1-y)$.

Note. Although the functions introduced above are point indexed, we will use the same symbol to represent their corresponding set-indexed functions whenever they are well defined. For example, since $K(\cdot, s)$ is nondecreasing, 
it determines a Lebesgue-Stieltjes measure which we will write as $K(B, s)$. In particular, (1.8) implies

$$
E K(s J, s)=\frac{2}{s}|J|
$$

for any Borel subset $J$ of $[0,1]$ and $s<1$. Here, $|J|$ denotes the Lebesgue measure of $J$.

The outline of the paper is as follows. The key result about the eventual simple form of the cumulants is proved in Section 2. The weak convergence of the empirical processes for the division points and for the normalized spacings are obtained, respectively, in Sections 4 and 6 . The corresponding preliminary results for the convergence of the stopped processes are given, respectively, in Sections 3 and 5. Finally, in Section 7, the covariance function for the limiting Gaussian processes in the spacings case is derived, thereby characterizing those processes completely.

2. Cumulants of functions of the stopped process. As mentioned above, there is a fundamental recursive structure present in the Kakutani interval-splitting procedure that is central to its study. Recall that $N_{s}$ is the number of partition points that are necessary to get all spacings $\leq s$. The first splitting point, $X_{1}$, is a $\operatorname{Unif}(0,1)$ r.v. For simplicity, write $U=X_{1}$. After the first split, there are two intervals, $(0, U)$ and $(U, 1)$ of lengths $U$ and $1-U$, respectively. Once $U$ is observed, the procedure is equivalent to watching two independent Kakutani procedures taking place on these two intervals until both of them result in spacings smaller than $s$. Moreover, the number of division points needed to partition an interval of length $U$ according to the $K$-model until no subinterval exceeds $s$ has the same distribution as the number of points needed to divide $(0,1)$ so that no subinterval exceeds $s / U$. From this, the representations (1.9) and (1.10) follow. These relations are really of the same type. For if one sets $x=y t$ in (1.10), then for fixed $y$, the resulting recursion for $K$ is of the same form as that which (1.9) gives for $N_{t}+1$. To emphasize this general nature, let $\{D(t): t>0\}$ be a realvalued process satisfying

$$
D(t) \stackrel{L}{=} D(t / U)+D^{*}(t /(1-U)) \quad \text { for } 0<t<1
$$

where $D={ }_{L} D^{*}, U$ is $\operatorname{Unif}(0,1)$ and $D, D^{*}$ and $U$ are independent.

LEMMA 2.1. If $D$ satisfies (2.1) and, for a positive integer $m, E|D(t)|^{m}$ is bounded for $t \geq 1$, then for every $t_{0}>0, E|D(t)|^{m}$ is bounded for $t \geq t_{0}$.

ProOF. Fix a positive integer $r$ and choose $t \in\left[2^{-r}, 2^{-r+1}\right.$ ). Define independent $D_{0}, D_{1}, \ldots$ and $U_{1}, U_{2}, \ldots$ with $D_{i}={ }_{L} D$ and $U_{i}={ }_{L} U$ for all $i$. Let 
$V_{i}=U_{i} \vee\left(1-U_{i}\right), W_{i}=U_{i} \wedge\left(1-U_{i}\right)$ and

$$
v_{t}=\min \left\{n: \prod_{i=1}^{n} V_{i} \leq t 2^{r-1}\right\} .
$$

By iterating (2.1) until the arguments of the $D_{i}$ are all $\geq 2^{-r+1}$ we find

$$
\begin{aligned}
D(t) \stackrel{L}{=} & D_{0}\left(t / W_{1}\right)+D_{1}\left(t / V_{1}\right) \\
\underline{L} & D_{0}\left(t / W_{1}\right)+\mathbb{1}_{\left\{v_{t}=1\right\}} D_{1}\left(t / V_{1}\right) \\
& \quad+\mathbb{1}_{\left\{v_{t}>1\right\}}\left[D_{1}\left(t /\left(V_{1} W_{2}\right)\right)+D_{2}\left(t /\left(V_{1} V_{2}\right)\right)\right] \\
\underline{L} & \cdots \stackrel{L}{=} \sum_{k=0}^{v_{t}-1} D_{k}\left(t /\left(\left(\prod_{i=1}^{k} V_{i}\right) W_{k+1}\right)\right)+D_{v_{t}}\left(t / \prod_{i=1}^{v_{t}} V_{i}\right) .
\end{aligned}
$$

Conditioning first on $\left\{U_{1}, U_{2}, \ldots\right\}$ (and hence on $v_{t}$ ), Minkowski's inequality implies

$$
E|D(t)|^{m} \leq E\left(v_{t}+1\right)^{m} \sup \left\{E|D(s)|^{m}: s \geq 2^{-r+1}\right\} .
$$

Now Markov's inequality yields

$$
\begin{aligned}
P\left(v_{t}>n\right) & =P\left(\prod_{i=1}^{n} V_{i}>t 2^{r-1}\right) \leq P\left(\prod_{i=1}^{n} V_{i}>\frac{1}{2}\right) \\
& \leq 2 E \prod_{i=1}^{n} V_{i}=2\left(\frac{3}{4}\right)^{n},
\end{aligned}
$$

so that

$$
E\left(v_{t}+1\right)^{m}=\sum_{n=2}^{\infty} n^{m} P\left(v_{t}=n-1\right) \leq 2 \sum_{n=2}^{\infty} n^{m}(3 / 4)^{n-2}=A_{m}<\infty .
$$

Because $A_{m}>1$, this yields

$$
\begin{aligned}
\sup \left\{E|D(t)|^{m}: t \geq 2^{-r}\right\} & \leq A_{m} \sup \left\{E|D(t)|^{m}: t \geq 2^{-r+1}\right\} \\
& \leq A_{m}^{r} \sup \left\{E|D(t)|^{m}: t \geq 1\right\}
\end{aligned}
$$

by recursion over $r$, which gives the desired result.

We now establish that the structure of $D$ that is implicit in the representation (2.1) forces the process $D$ to have a pseudo-Poissonian nature (in terms of cumulants) as is made precise in the following theorem. Here and throughout, we denote the $m$ th cumulant and the $m$ th central moment of a r.v. $Z$ by $\kappa_{m}(Z)$ and $\mu_{m}(Z)$, respectively. 
TheOREM 2.2. Suppose that $D$ satisfies (2.1) and that, for $m=1,2, \ldots$, $E|D(t)|^{m}$ is bounded for $t \geq 1$. There then exist constants $c_{1}, c_{2}, \ldots$ such that

$$
E D(t)=\frac{c_{1}}{t} \quad \text { for } 0<t<1,
$$

and for $m \geq 2$,

$$
\kappa_{m}(D(t))=\frac{c_{m}}{t} \quad \text { for } 0<t \leq 1 / m .
$$

It follows that $c_{1}=\lim _{t \uparrow 1} E D(t)$ and $c_{m}=m^{-1} \kappa_{m}(D(1 / m))$.

Proof. We write $\kappa_{m}(t)=\kappa_{m}(D(t))$ and $\mu(t)=\kappa_{1}(t)=E D(t)$. For $0<t<1,(2.1)$ implies that

$$
\mu(t)=\int_{0}^{1}\{\mu(t / u)+\mu(t /(1-u))\} d u=2 \int_{0}^{1} \mu(t / u) d u=2 t \int_{t}^{\infty} \frac{\mu(y)}{y^{2}} d y .
$$

By Lemma 2.1, $\sup \{\mu(y): y \geq t\}$ is bounded for $t>0$, so that $\mu$ is first of all continuous on $(0,1)$, and therefore also differentiable on $(0,1)$ with

$$
\left(\frac{\mu(t)}{t}\right)^{\prime}=\frac{\mu^{\prime}(t)}{t}-\frac{\mu(t)}{t^{2}}=-2 \frac{\mu(t)}{t^{2}} .
$$

Hence $\mu(t)+t \mu^{\prime}(t)=0$ on $(0,1)$ and (2.2) follows.

Define

$$
\psi(t, w)=\log (E \exp \{i w D(t)\})=\sum_{j=1}^{\infty} \kappa_{j}(t) \frac{(i w)^{j}}{j !} .
$$

The right-hand side is an asymptotic expansion in the sense that if we truncate the sum after $r$ terms, the remainder is $O\left(|w|^{r+1}\right)$ as $w \rightarrow 0$, uniformly for $t \geq t_{0}>0$. Of course (2.1) implies

$$
\begin{aligned}
\exp \{\psi(t, w)\} & =\int_{0}^{1} \exp \{\psi(t / u, w)+\psi(t /(1-u), w)\} d u \\
& =2 \int_{0}^{1 / 2} \exp \{\psi(t / u, w)+\psi(t /(1-u), w)\} d u .
\end{aligned}
$$

Fix $m \geq 2, t \in(0,1 / m]$, and assume that $\kappa_{j}(t)=c_{j} / t$ for $t<1 / j$ and $j=$ $1,2, \ldots, m-1$. To prove (2.3) we shall show that this implies that $\kappa_{m}(t)=c_{m} / t$ for $t \leq 1 / m$. Define $n=[m / 2]$ and note that:

(i) if $u \in(n t, 1 / 2)$, then $t /(1-u)<t / u<1 / n$, so that $\kappa_{j}(t / u)=c_{j} u / t$ and $\kappa_{j}(t /(1-u))=c_{j}(1-u) / t$ for $j=1,2, \ldots, n$;

(ii) if $u \in((k-1) t, k t)$ for some $k=1, \ldots, n$, then $t / u<1 /(k-1)$ and $t /(1-u)<1 /(m-k)$, so that $\kappa_{j}(t / u)=c_{j} u / t$ for $j=1, \ldots, k-1$ and $\kappa_{j}(t /(1-u))=c_{j}(1-u) / t$ for $j=1, \ldots, m-k$. 
Multiplying (2.4) by

$$
\exp \left\{-\sum_{j=1}^{m-1} \kappa_{j}(t) \frac{(i w)^{j}}{j !}\right\}=\exp \left\{-t^{-1} \sum_{j=1}^{m-1} c_{j} \frac{(i w)^{j}}{j !}\right\}
$$

we find

$$
\begin{array}{r}
\exp \left\{\sum_{j=m}^{\infty} \kappa_{j}(t) \frac{(i w)^{j}}{j !}\right\} \\
=2 \int_{n t}^{1 / 2} \exp \left\{\sum_{j=n+1}^{\infty}\left(\kappa_{j}\left(\frac{t}{u}\right)+\kappa_{j}\left(\frac{t}{1-u}\right)\right) \frac{(i w)^{j}}{j !}-\frac{1}{t} \sum_{j=n+1}^{m-1} c_{j} \frac{(i w)^{j}}{j !}\right\} d u \\
+2 \sum_{k=1}^{n} \int_{(k-1) t}^{k t} \exp \left\{\sum_{j=k}^{m-k}\left(\kappa_{j}\left(\frac{t}{u}\right)-c_{j} \frac{u}{t}\right) \frac{(i w)^{j}}{j !}\right. \\
\quad+\sum_{j=m-k+1}^{m-1}\left(\kappa_{j}\left(\frac{t}{u}\right)+\kappa_{j}\left(\frac{t}{1-u}\right)-\frac{c_{j}}{t}\right) \frac{(i w)^{j}}{j !}
\end{array}
$$

Now we expand both sides in powers of $(i w)$ and equate the coefficients of $(i w)^{m} / m$ !. Note that in the first integral only terms containing $\kappa_{m}$ contribute to this coefficient and that

$$
\int_{(k-1) t}^{k t} f(t / u) d u=t \int_{k-1}^{k} f(1 / y) d y=C t
$$

where $C=C(k, f)$ is constant in $t$. Hence we find after some reflection that

$$
\begin{aligned}
\kappa_{m}(t) & =\int_{0}^{1}\left(\kappa_{m}(t / u)+\kappa_{m}(t /(1-u))\right) d u+C t=2 \int_{0}^{1} \kappa_{m}(t / u) d u+C t \\
& =2 t \int_{t}^{\infty} \kappa_{m}(y) y^{-2} d y+C t \quad \text { for } 0<t \leq 1 / m .
\end{aligned}
$$

By Lemma 2.1, $\kappa_{m}(y)$ is bounded on $(t, \infty)$, so that $\kappa_{m}$ is continuous on $(0,1 / m)$ and differentiable on $(0,1 / m)$ with

$$
\left(\frac{\kappa_{m}(t)}{t}\right)^{\prime}=\frac{\kappa_{m}^{\prime}(t)}{t}-\frac{\kappa_{m}(t)}{t^{2}}=-2 \frac{\kappa_{m}(t)}{t^{2}}
$$

It follows that $\kappa_{m}(t)+t \kappa_{m}^{\prime}(t)=0$ on $(0,1 / m)$ and so $\kappa_{m}(t)=c_{m} / t$ for $t \in$ $(0,1 / m]$.

In view of (1.9) and (1.10), two special examples of $D$-processes to which this theorem applies are $N_{t}+1$ and $K(\alpha t, t)$. Since these examples are central in what follows, we summarize their structure as follows. 
COROLlary 2.3. For $m=2,3, \ldots$ and $0<t \leq 1 / m$, the cumulants of $N_{t}$ and $K(\alpha t, t)$ for $0<\alpha \leq 1$ are given by

$$
\begin{aligned}
\kappa_{m}\left(N_{t}\right)=\kappa_{m}\left(N_{t}+1\right) & =c_{m} / t \quad \text { with } c_{m}=\frac{1}{m} \kappa_{m}\left(N_{1 / m}\right), \\
\kappa_{m}(K(\alpha t, t)) & =c_{m, \alpha} / t \quad \text { with } c_{m, \alpha}=\frac{1}{m} \kappa_{m}(K(\alpha / m, 1 / m))
\end{aligned}
$$

and $E\left(N_{t}\right)=\mu(t)=2 / t-1, E(K(\alpha t, t))=2 \alpha / t$ for $0<t<1$.

In particular, this corollary shows that the variance of $N_{t}$ is $c / t$ if $0<t \leq 1 / 2$ (with $c=c_{2}$ ), as given previously in (1.7), and the fourth central moment is

$$
\mu_{4}(t):=E\left[N_{t}-\mu(t)\right]^{4}=c_{4} / t+3 c^{2} / t^{2} \quad \text { if } 0<t \leq 1 / 4 .
$$

The latter is needed several times in what follows.

The main result above generalizes straightforwardly to the case of vectorvalued $\mathbf{D}(t)=\left(D_{1}(t), D_{2}(t), \ldots, D_{r}(t)\right)$. In this paper, only the bivariate case $r=2$, is needed (in Sections 5 and 7) so we will restrict our discussion to this case for notational convenience. In analogy with the univariate case, multivariate cumulants are the coefficients in the multivariate Taylor expansion of the logarithm of the joint characteristic function. Thus in particular, if $Z=(X, Y)$ is a r.v. with $E|X|^{m}|Y|^{n}<\infty$ for all $m, n \geq 1$, the $(m, n)$ th cumulants, $\kappa_{m, n} \equiv \kappa_{m, n}(X, Y)$ are defined by

$$
\log E \exp (i v X+i w Y)=\sum_{\substack{m=0 \\ m+n \geq 1}}^{\infty} \sum_{n=0}^{\infty} \kappa_{m n} \frac{(i v)^{m}}{m !} \frac{(i w)^{n}}{n !} .
$$

Clearly, the joint cumulants $\left\{\kappa_{m n}\right\}$ are determined by the univariate cumulants of $v X+w Y$; for $l \geq 1$,

$$
\kappa_{l}(v X+w Y)=\sum_{m=0}^{l}\left(\begin{array}{c}
l \\
m
\end{array}\right) v^{m} w^{l-m} \kappa_{m, l-m} .
$$

Now, if we take $X=D_{1}(t), Y=D_{2}(t)$ and assume that for every $v, w, v D_{1}(t)+$ $w D_{2}(t)$ satisfies the conditions of Theorem 2.2 so that

$$
\kappa_{l}(t) \equiv \kappa_{l}\left(v D_{1}(t)+w D_{1}(t)\right)=\frac{c_{l}(v, w)}{t} \quad \text { for } 0<t \leq 1 / l,
$$

it follows from the identity in (2.7) that the coefficients $\kappa_{m, l-m}(t)$, now depending upon $t$, must satisfy $\kappa_{m, l-m}(t)=c_{m, l-m} / t$ for $0<t \leq 1 / l$ for some constants $c_{m, l-m}$. This verifies:

THEOREM 2.4. Let $\mathbf{D}(t)=\left(D_{1}(t), D_{2}(t)\right), t>0$, satisfy (2.1), with $E\left|D_{1}(t)\right|^{m}$ and $E\left|D_{2}(t)\right|^{m}$ bounded in $t \geq 1$ for each $m \geq 1$. Then there exist constants $\left\{c_{m n}\right\}$ such that

$$
E D_{1}(t)=\frac{c_{10}}{t}, \quad E D_{2}(t)=\frac{c_{01}}{t} \quad \text { for } 0<t<1
$$


and for $m \geq 0, n \geq 0, m+n \geq 2$,

$$
\kappa_{m n}(\mathbf{D}(t))=\frac{c_{m, n}}{t} \quad \text { for } 0<t \leq \frac{1}{m+n} .
$$

Note that in the above, $\kappa_{10}(\mathbf{D}(t))=\kappa_{1}\left(D_{1}(t)\right)=E D_{1}(t)$, with a similar identity for $D_{2}$.

3. Weak convergence of the $\boldsymbol{U}(\cdot, s)$ processes. In this section, we prove that the stopped empirical process of the division points, $U(\cdot, s)$, as defined in (1.15), converges weakly to a nonstandard Brownian bridge, $\sigma B_{0}$, as $s \rightarrow 0$ in which the constant $\sigma=(4 \ln 2-5 / 2)^{1 / 2} \approx 0.5221003$. [It turns out that $\sigma^{2}=c / 2$ with $c$ defined in (1.7).] In the following section, we show that $U_{n}$, the ordinary empirical process for the partition points, inherits this same limit. Consequently, even though the $K$-model is indistinguishable from the $U$-model with regard to the Glivenko-Cantelli result for division points, when one considers weak convergence the two cases are quite different. The $K$-model results in a limiting process that has only about half of the variation as does the limit under the $U$-model.

Consider the definitions of the empirical processes of partition points given in (1.15). With $U_{n}=n^{1 / 2}\left(F_{n}-F\right)$, the stopped version of the process would be $U_{N_{s}}$. But

$$
\begin{aligned}
U_{N_{s}}(x) & =\left(N_{s}\right)^{1 / 2}\left\{\frac{N_{s}(x)}{N_{s}}-x\right\}, \quad 0 \leq x \leq 1,0<s<1, \\
& =\left(N_{s}\right)^{-1 / 2}\left\{N_{s}(x)-x N_{s}\right\} .
\end{aligned}
$$

Since $s N_{s} \rightarrow 2$ a.s. by (1.11), this process is asymptotically equivalent to $U(x, s)$. But one can expand

$$
\begin{aligned}
U(x, s) & =(s / 2)^{1 / 2}\left\{N_{s}(x)-x N_{s}\right\} \\
& =(s / 2)^{1 / 2}\left\{N_{s}(x)-\frac{2 x}{s}\right\}-x(s / 2)^{1 / 2}\left\{N_{s}-\frac{2}{s}\right\} .
\end{aligned}
$$

Just as for the usual $U$-model, this representation suggests the study of the nontied-down process

$$
Z(x, s):=(s / 2)^{1 / 2}\left\{N_{s}(x)-2 x / s\right\}, \quad 0 \leq x \leq 1,0<s<1,
$$

in terms of which $U(x, s)=Z(x, s)-x Z(1, s)$. The proof of the following theorem is therefore a proof of the convergence of $Z(\cdot, s)$, from which that of $U(\cdot, s)$ follows directly.

THEOREM 3.1. As $t \rightarrow 0, U(\cdot, t) \rightarrow_{L} \sigma B_{0}(\cdot)$, where $B_{0}$ is standard Brownian bridge and $\sigma^{2}=\frac{1}{4} \operatorname{var}\left(N_{1 / 2}\right)=4 \ln 2-5 / 2$ so that $\sigma=0.5221003$. 
PROOF. For $0<s<1$, introduce the notation $0=X_{s 0} \leq X_{s 1} \leq \cdots \leq X_{s, N_{s}} \leq$ $X_{s, N_{s}+1}=1$ to represent the $N_{s}$ division points and write $D_{s i}=D_{N_{s} i}=X_{s i}-$ $X_{s, i-1}, 1 \leq i \leq N_{s}+1$, for the associated spacings. For $x \in[0,1]$, define $X_{s}(x)=$ $X_{s, N_{s}(x)}$ and $X_{s}^{+}(x)=X_{s, N_{s}(x)+1}$, so that $X_{s}(x) \leq x<X_{s}^{+}(x)$ are the division points that straddle $x$. Write $D_{s}(x)=X_{s}^{+}(x)-X_{s}(x)$.

The following representation is key. For any $0<t<s<1$,

$$
N_{t}(x) \stackrel{L}{=} \sum_{i=1}^{N_{s}(x)}\left(N_{t / D_{s i}}^{(i)}+1\right)+N_{t / D_{s}(x)}^{*}\left(\frac{x-X_{s}(x)}{D_{s}(x)}\right)
$$

where $\left\{N_{.}^{(i)}\right\}$ are independent processes with the same laws as $N_{.}, N_{.}^{*}(\cdot)={ }_{L} N_{.}(\cdot)$ and all of these processes are independent of each other and of $N_{s}(\cdot)$. Thus, conditionally given $\mathcal{F}_{s}=\sigma\left(D_{s i}: 1 \leq i \leq N_{s}+1\right)=\sigma\left(X_{1}, X_{2}, \ldots, X_{N_{s}}\right), N_{t}(x)$ is a sum of independent r.v.'s. More to the point is the observation that $N_{t}(\cdot)$ is essentially a partial-sum process, the difference being the $N^{*}$-term in (3.2).

Our approach, suggested by (3.2), is to apply standard weak convergence results to this partial-sum process, and then show that the difference term is negligible. Actually, there are two partial-sum processes involved. The one suggested by (3.2) has jumps of $N_{t / D_{s i}}^{(i)}+1$ at the times $X_{s i}$. [Remember that we will be studying these processes conditional on $\mathcal{F}_{s}$ and with $t=t(s)<s$ going to zero appropriately with $s$.] The more standard time scale for plotting partial-sum processes is to plot the $i$ th sum at its variance. We will therefore first use this standard time scale to get weak convergence, then show that the difference between the two time scales converges uniformly to zero, and finally prove that the contribution due to the extra $N^{*}$ term in (3.2) is negligible.

Write

$$
S_{t}(x ; s):=(t / 2)^{1 / 2} \sum_{i: X_{s i} \leq x}\left\{N_{t / D_{s i}}^{(i)}-\mu\left(t / D_{s i}\right)\right\},
$$

where $\mu(s)=E N_{s}$ is given in (1.6). Thus $S_{t}(\cdot ; s)$ is a partial sum process with increments $(t / 2)^{1 / 2}\left[N_{t / D_{s i}}^{(i)}-\mu\left(t / D_{s i}\right)\right]$ plotted at $X_{s i}$. Let $S_{t}^{*}(\cdot ; s)$ be the related partial-sum process whose increments are the same but which are plotted at the cumulative proportional variances $\tau_{i}=\left(\sigma_{1}^{2}+\cdots+\sigma_{i}^{2}\right) /\left(\sigma_{1}^{2}+\cdots+\sigma_{N_{s}+1}^{2}\right)$ with $\sigma_{i}^{2}=\operatorname{var} N_{t / D_{s i}}^{(i)}$. Before obtaining the limit of this $S_{t}^{*}(\cdot ; s)$ process it is necessary to determine the limiting behavior of the time scale given by $\left\{\tau_{i}\right\}$. For this, we first need to complete the evaluation of $v(u)=\operatorname{var}\left(N_{u}\right)$.

From (1.7) and the definition of $N_{u}$, it is known that $v(u)=0$ if $u \geq 1$ and $=c / u$ for $0<u \leq 1 / 2$ where $c=v(1 / 2) / 2$. It remains to compute $v(u)$ for $1 / 2 \leq u<1$ and thereby evaluate $c$.

LEMMA 3.2. For $1 / 2 \leq u<1$, the distribution of $N_{u}$ is given by

$$
P\left[N_{u}>k\right]=2^{k} u \sum_{j=k}^{\infty}\left(\ln \frac{1}{u}\right)^{j} / j !=2^{k} P\left[\mathcal{P}\left(\ln \frac{1}{u}\right) \geq k\right]
$$


for $k=0,1,2, \ldots$ where $\mathcal{P}(\lambda)$ denotes a Poisson r.v. of mean $\lambda$. Moreover, the variance of $N_{u}$ is

$$
v(u)=\frac{1}{u}\left(8 \ln \frac{1}{u}+2\right)-\frac{4}{u^{2}}+2, \quad \frac{1}{2} \leq u<1 .
$$

In particular, $c=v(1 / 2) / 2=8 \ln 2-5$.

ProOF. For $1 / 2 \leq u<1$, the splitting points $X_{1}, X_{2}, \ldots, X_{N_{u}}$ never return to an interval they have left, so that as in the proof of Lemma 2.1,

$$
P\left[N_{u}>k\right]=P\left[\prod_{i=1}^{k} V_{i}>u\right]
$$

where $V_{1}, V_{2}, \ldots$ are independent $\operatorname{Unif}(1 / 2,1)$ r.v.'s. Hence the $-\ln V_{i}$ are distributed as independent standard exponential random variables $Z_{i}$, each conditioned on being smaller than $\ln 2$. For $1 / 2 \leq u<1, \sum_{i=1}^{k} Z_{i}<\ln (1 / u)$ implies $Z_{i}<\ln (1 / u) \leq \ln 2$ for $i=1, \ldots, k$ and

$$
\begin{aligned}
P\left[N_{u}>k\right] & =P\left(\sum_{i=1}^{k} Z_{i}<\ln \frac{1}{u} \mid \max Z_{i}<\ln 2\right) \\
& =2^{k} P\left(\sum_{i=1}^{k} Z_{i}<\ln \frac{1}{u}\right) \\
& =2^{k} P\left(\mathcal{P}\left(\ln \frac{1}{u}\right) \geq k\right),
\end{aligned}
$$

which proves (3.4). This in turn implies

$$
\begin{aligned}
E N_{u}^{2} & =\sum_{k=0}^{\infty}(2 k+1) P\left[N_{u}>k\right]=u \sum_{j=0}^{\infty} \frac{(\ln 1 / u)^{j}}{j !} \sum_{k=0}^{j}(2 k+1) 2^{k} \\
& =u \sum_{j=0}^{\infty} \frac{(\ln 1 / u)^{j}}{j !}\left\{(2 j-1) 2^{j+1}+3\right\} \\
& =\frac{1}{u}\left(8 \ln \frac{1}{u}-2\right)+3 .
\end{aligned}
$$

Since $E N_{u}=2 / u-1$ by (1.6), the expression (3.5) follows by direct calculation.

To establish the weak convergence of the $S_{t}^{*}(\cdot, s)$ partial-sum process, it suffices [cf. Gihman and Skorokhod (1974), page 411] to show [with $E_{S}$ and $P_{S}$ denoting the conditional quantities, $E\left(-\mid \mathcal{F}_{S}\right)$ and $P\left[-\mid \mathcal{F}_{S}\right]$, respectively, that

$$
\lim _{s \rightarrow 0} \sum_{i=1}^{N_{s}+1} E_{s} t\left\{N_{t / D_{s i}}^{(i)}-\mu\left(t / D_{s i}\right)\right\}^{2} \mathbb{1}_{\left[t^{1 / 2}\left|N_{t / D_{s i}}^{(i)}-\mu\left(t / D_{s i}\right)\right|>\varepsilon\right]}=0,
$$


for a suitable choice of $t=t(s)<s$ going to zero with $s$. By the Cauchy-Schwarz and Chebyshev inequalities, the sum in (3.6) is bounded by

$$
\sum_{i=1}^{N_{s}+1} t\left\{\mu_{4}\left(t / D_{s i}\right) t v\left(t / D_{s i}\right) \varepsilon^{-2}\right\}^{1 / 2}
$$

in which $\mu_{4}(u)=E\left[N_{u}-\mu(u)\right]^{4}$ and $v(u)=\operatorname{var}\left(N_{u}\right)$. By definition and by Theorem 2.2, $u^{2} \mu_{4}(u)$ and $u v(u)$ are bounded for all $u>0$. Hence the bound in (3.7) is

$$
\varepsilon^{-1} \sum_{i=1}^{N_{s}+1} D_{s i}^{3 / 2}\left\{\left(t / D_{s i}\right)^{2} \mu_{4}\left(t / D_{s i}\right)\right\}^{1 / 2}\left\{\left(t / D_{s i}\right) v\left(t / D_{s i}\right)\right\}^{1 / 2} \leq C_{0}\left(M_{N_{s}}\right)^{1 / 2}
$$

for some constant $C_{0}$ where $M_{n}$ is the maximum spacing at the $n$th stage. But by (1.13) this bound goes to zero, which establishes (3.6) and hence the desired weak convergence result. The limit process must be a mean zero Brownian motion and it remains only to determine its variance at $x=1$. By the discussion following (3.3),

$$
(t / 2)^{1 / 2} \sum_{i=1}^{N_{s}+1}\left\{N_{t / D_{s i}}^{(i)}-\mu\left(t / D_{s i}\right)\right\}=S_{t}^{*}(1, s) .
$$

By (1.7) and (3.5) the (conditional) variance of this sum, with $\sigma^{2}=c / 2$, is equal to

$$
\begin{aligned}
\frac{t}{2} \sum_{i=1}^{N_{s}+1} v\left(t / D_{s i}\right) & =t \sum_{i: D_{s i}>2 t} \sigma^{2} D_{s i} / t+\frac{t}{2} \sum_{i: t<D_{s i} \leq 2 t} v\left(t / D_{s i}\right) \\
& =\sigma^{2}+\frac{t}{2} \sum_{i: D_{s i} \leq 2 t}\left\{v\left(t / D_{s i}\right)-2 \sigma^{2} D_{s i} / t\right\} \\
& =\sigma^{2}+\frac{1}{2} \sum_{i: D_{s i} \leq 2 t} D_{s i}\left\{\left(t / D_{s i}\right) v\left(t / D_{s i}\right)-2 \sigma^{2}\right\} .
\end{aligned}
$$

But since $u v(u)$ is bounded for all $u>0$ (see Theorem 2.2) and since $\Sigma\left\{D_{s i}: D_{s i} \leq\right.$ $2 t\} \leq 2 t K(2 t, s)$, the second term in (3.9) is bounded for some constant $C$ by $C t K(2 t, s)=C\left(\frac{t}{s}\right) s K\left(\frac{2 t}{s} s, s\right)$. By (1.14) this is $O(1)$ with probability 1 and, moreover, is $o(1)$ if $t=o(s)$. This proves that when $t / s \rightarrow 0$, var $S_{t}^{*}(1, s) \rightarrow \sigma^{2}=$ $c / 2=v(1 / 2) / 4=4 \ln 2-5 / 2$ by Lemma 3.2. Thus

$$
S_{t}^{*}(\cdot, s) \stackrel{L}{\rightarrow} \sigma B(\cdot)
$$

as $s \rightarrow 0$ with $t=o(s)$. 
REMARK. Let us clarify how the unconditional weak convergence follows from conditional applications of limit theorems. Our approach is to use two parameter values, $t<s$ with $t=t(s)$, and express a process, $X_{t}(\cdot)$ say, in such a way that conditionally given $\widetilde{F}_{s}$ a limit result holds. For example, if $g$ is any bounded continuous real-valued function defined on the range of $X_{t}(\cdot)$, suppose $E\left\{g\left(X_{t}(\cdot)\right) \mid \mathcal{F}_{s}\right\} \rightarrow E g(X(\cdot))$ a.s. Then by Lebesgue's dominated convergence theorem, $E g\left(X_{t}(\cdot)\right) \rightarrow E g(X(\cdot))$. This example suffices for our purposes, since it shows how conditional weak convergence a.s. proves unconditional weak convergence; all of our examples are for $D[0,1]$ processes with limits in $C(0,1)$.

We now compare the time scales of $S_{t}(\cdot ; s)$ and $S_{t}^{*}(\cdot ; s)$. By (3.3), the increments of $S_{t}(\cdot ; s)$ are the same as for $S_{t}^{*}(\cdot ; s)$ but they occur at $X_{s i}$ rather than at $\tau_{i}$. The differences between the two time scales are

$$
X_{s j}-\tau_{j}=\sum_{i=1}^{j}\left\{D_{s i}-v\left(t / D_{s i}\right) / \bar{\sigma}_{s}^{2}\right\}
$$

where $\bar{\sigma}_{s}^{2}=\sigma_{1}^{2}+\cdots+\sigma_{N_{s}+1}^{2}$. Note that $t \bar{\sigma}_{s}^{2} / 2$ is equal to (3.9) and therefore $t \bar{\sigma}_{s}^{2} / 2=\sigma^{2}+o(1)$ a.s. if $t=o(s)$, as was shown following (3.9).

By means of the same partition used in (3.9),

$$
\begin{aligned}
\max _{1 \leq j \leq N_{s}+1}\left|X_{s j}-\tau_{j}\right| \leq\left(t \bar{\sigma}_{s}^{2}\right)^{-1} t \sum_{i=1}^{N_{s}+1}\left|\bar{\sigma}_{s}^{2} D_{s i}-v\left(\frac{t}{D_{s i}}\right)\right| \\
\leq\left(t \bar{\sigma}_{s}^{2}\right)^{-1}\left\{\left|t \bar{\sigma}_{s}^{2}-2 \sigma^{2}\right|+2 \sigma^{2} \sum_{D_{s i} \leq t} D_{s i}\right. \\
\left.\quad+\sum_{t<D_{s i} \leq 2 t} D_{s i}\left|2 \sigma^{2}-\left(\frac{t}{D_{s i}}\right) v\left(\frac{t}{D_{s i}}\right)\right|\right\} \\
\leq o(1)+C_{0} 2 t K(2 t, s)
\end{aligned}
$$

for some constant $C_{0}$. Thus as before, (1.14) implies that this converges a.s. to zero as $s \rightarrow 0$ provided $t=o(s)$. Since this proves that the difference between the time scales converges uniformly to zero with probability 1, it follows from (3.10) and the above remark that

$$
S_{t}(\cdot ; s) \stackrel{L}{\rightarrow} \sigma B(\cdot)
$$

as $s \rightarrow 0$ with $t=o(s)$.

It remains to show that the extra $N^{*}$ term in (3.2) and the centering differences of the $Z(\cdot, t)$ and $S_{t}(\cdot ; s)$ processes are asymptotically negligible. Observe first that the $N^{*}$ term is bounded by

$$
\sup _{0 \leq x \leq 1}\left|N_{t}(x)-\sum_{i=1}^{N_{s}(x)}\left(N_{t / D_{s i}}^{(i)}+1\right)\right|=\max _{1 \leq i \leq N_{s}+1}\left\{N_{t / D_{s i}}^{(i)}+1\right\}:=M_{s, t}
$$


[The difference on the left-hand side is actually nonnegative by (3.2) and approaches $N_{t / D_{s, i+1}}^{(i)}+1$ as $x \nearrow X_{s, i+1}$.] To show that $t^{1 / 2} M_{s, t} \rightarrow 0$ in probability, given $\mathcal{F}_{s}$, compute

$$
\begin{aligned}
P_{s}\left[M_{s, t}>\varepsilon t^{-1 / 2}\right] & \leq \sum_{i=1}^{N_{s}+1} P_{s}\left[N_{t / D_{s i}}^{(i)}+1>\varepsilon t^{-1 / 2}\right] \\
& \leq\left(N_{s}+1\right) P\left[N_{t / s}+1>\varepsilon t^{-1 / 2}\right]
\end{aligned}
$$

since each $D_{s i} \leq s$. Thus in particular, each $N_{t / D_{s i}}^{(i)}$ is stochastically (given $\mathcal{F}_{s}$ ) less than $N_{t / s}$. Suppose $t / s \leq 1 / 4$. Then by (2.6), Markov's inequality with fourth moments gives

$$
\begin{aligned}
P\left[N_{t / s}+1>\varepsilon t^{-1 / 2}\right] & \leq \mu_{4}(t / s) /\left(\varepsilon t^{-1 / 2}-2 s / t\right)^{4} \\
& =\left\{c_{4} s / t+12 \sigma^{4} s^{2} / t^{2}\right\} /\left(\varepsilon t^{-1 / 2}-2 s / t\right)^{4}
\end{aligned}
$$

so that

$$
P_{s}\left[M_{s, t}>\varepsilon t^{-1 / 2}\right] \leq s\left(N_{s}+1\right) \frac{c_{4} t+12 \sigma^{4} s}{\left(\varepsilon-2 s / t^{1 / 2}\right)^{4}} .
$$

Since $s N_{s} \rightarrow 2$ a.s. by (1.11), this bound converges to zero a.s. provided $s=$ $o(\sqrt{t})$. In view of (3.12) we also need $t=o(s)$, so choose $t=s^{3 / 2}$. In this case then, this proves that almost surely, $t^{1 / 2} M_{s, t} \rightarrow 0$ in probability conditionally given $\mathcal{F}_{S}$.

Now by (3.1)-(3.3) and (3.13),

$$
\begin{aligned}
& \sup _{0 \leq x \leq 1}\left|Z(x, t)-S_{t}(x ; s)\right| \\
& \quad \leq t^{1 / 2} M_{s, t}+t^{1 / 2} \sum_{i=1}^{N_{s}}\left|\mu\left(t / D_{s i}\right)+1-2 D_{s i} / t\right|+2 t^{-1 / 2} M_{N_{s}}
\end{aligned}
$$

where we have written $x=\sum_{i=1}^{N_{s}(x)} D_{s i}+\left(x-X_{s i}\right)$ and used $\left|x-X_{s i}\right| \leq D_{s}(x)$ and so $\max _{i}\left|x-X_{s i}\right| \leq \max _{i} D_{s i}=M_{N_{s}}$. We have just shown that the first term is $o_{p}(1)$ a.s. if $s=o(\sqrt{t})$. By (1.13) the third term is $o(1)$ under the same proviso. By (1.6) the middle term is bounded by

$$
t^{1 / 2} \sum_{i: D_{s i} \leq t}\left|2 D_{s i} / t-1\right| \leq t^{1 / 2} K(t, s) .
$$

Since $t<s<1, E\left\{t^{1 / 2} K(t, s)\right\}=2 t^{3 / 2} s^{-2}$ by (1.8). Thus if $t=o\left(s^{4 / 3}\right)$ this converges to zero and so the middle term on the right-hand side of (3.14) converges to zero in probability. We have thus established that if $s=s(t)=o(\sqrt{t})$ and $t=o\left(s^{4 / 3}\right)$, as is the case if $s=t^{2 / 3}$ for example, then the left-hand side of (3.14) is bounded by the sum of three terms, $T_{1}(t)+T_{2}(t)+T_{3}(t)$ say, in which 
$T_{2}$ and $T_{3}$ are measurable $\mathcal{F}_{s}$ and each converge in probability to zero, while with probability $1, T_{1}$ converges to zero in probability conditional on $\mathscr{F}_{s}$. It follows that $T_{1}$ also converges to zero in probability, thus showing that $Z(\cdot, t)$ and $S_{t}(\cdot ; s)$ converge weakly to the same limiting process when $s=t^{2 / 3}$. In view of (3.12) the proof of Theorem 3.1 is complete.

An important consequence of the above proof is that it gives the limiting distribution for $N_{t}$. Since $Z(1, t)=(t / 2)^{1 / 2}\left\{N_{t}-2 / t\right\}$, we have the following corollary.

COROllary 3.3. As $t \rightarrow 0,(t / 2)^{1 / 2}\left\{N_{t}-2 / t\right\}$ converges in law to a $N\left(0, \sigma^{2}\right)$ random variable, with $\sigma=4 \ln 2-5 / 2$ as in Theorem 3.1.

4. Weak convergence of the $\boldsymbol{U}_{\boldsymbol{n}}$ process. As discussed at the start of Section 3 , the stopped process $U(\cdot, s)$ is asymptotically equivalent to $U_{N_{s}}(\cdot)$, the regular empirical process of the division points computed at the random sample size $N_{s}$. We show in this section that the process $U_{n}$ inherits from $U(\cdot, s)$ and $U_{N_{s}}$ the same weak convergence. Thus the limiting process for $U_{n}$, which is $B_{0}$ under the $U$-model, becomes $\sigma B_{0}$ for the $K$-model.

Random sample size central limit theorems were first considered in a general setting by Anscombe (1952) who studied the case of sums of independent r.v.'s. The weak convergence of uniform empirical processes under random sample size was studied in Pyke (1968); see also Csörgó (1974) and Klaassen and Wellner (1992). The situation here is quite different in that we will deduce the convergence of the fixed sample size process from that of the random sample size case. Of course, one can reverse this formally by defining random times $s_{n}$ so that $U_{n}=U\left(\cdot, s_{n}\right)$; simply use $s_{n}=M_{n}$.

The result to be proved is the following:

TheOREM 4.1. As $n \rightarrow \infty, U_{n} \rightarrow_{L} \sigma B_{0}$, where $B_{0}$ is standard Brownian bridge and $\sigma=(4 \ln 2-5 / 2)^{1 / 2}=0.52210$.

PROOF. The proof essentially is by moments but entails a coupling argument in a critical spot. Some technical results are needed that are presented first in a series of lemmas. The first three involve the cumulants $\kappa_{m}$ and central moments $\mu_{m}$ of differences $N_{s}-N_{t}$.

LEMMA 4.2. For $m=2,3, \ldots$ and $0<s<t \leq 1 / m$,

$$
\kappa_{m}\left(N_{s}-N_{t}\right)=\kappa_{m}\left(N_{s / t m}-N_{1 / m}\right) \frac{1}{m t} .
$$

Proof. It is easy to check that $D(t)=N_{\alpha t}-N_{t}$ for $0<\alpha<1$ satisfies the relationship (2.1). Thus (4.1) follows from Theorem 2.2 with $\alpha=s / t$. 
LEMMA 4.3. For every $0<\varepsilon<1$ and $m=2,3, \ldots$, there exists a positive number $c_{m}(\varepsilon)$ such that

$$
\begin{aligned}
& \left|\kappa_{m}\left(N_{s}-N_{t}\right)\right| \leq c_{m}(\varepsilon)\left(\frac{1}{s}-\frac{1}{t}\right) \quad \text { for } \varepsilon t \leq s<t<1, \\
& \left|\mu_{m}\left(N_{s}-N_{t}\right)\right| \\
& \quad \leq c_{m}(\varepsilon)\left\{\left(\frac{1}{s}-\frac{1}{t}\right)^{m / 2}+\left(\frac{1}{s}-\frac{1}{t}\right)\right\} \quad \text { for } \varepsilon t \leq s<t<1 .
\end{aligned}
$$

Proof. First take $1 / m \leq t<1$. Let $M=K((s, t], t):=K(t, t)-K(s, t)$, be the number of intervals with length $l \in(s, t]$ at the first time when all intervals are less than or equal to $t$. Since $[M=0]=\left[N_{s}-N_{t}=0\right]$ and $N_{s}-N_{t} \geq 0$ a.s.,

$$
P(M>0)=P\left(N_{s}-N_{t} \geq 1\right) \leq E\left(N_{s}-N_{t}\right)=2(1 / s-1 / t) .
$$

Now, for $l \in(s, t], l / t \geq s / t \geq \varepsilon$ and since $M \leq 1 / s$ a.s.,

$$
N_{s}-N_{t} \leq \sum_{j=1}^{M} N_{s / t}^{(j)} \leq \mathbb{1}_{\{M>0\}} \sum_{j=1}^{[1 / s]} N_{\varepsilon}^{(j)}
$$

where the $N^{(j)}$ are independent copies of $N$ which are also independent of $M$. Hence, for $k=1,2, \ldots, m$ and $1 / m \leq t<1$, Minkowski's inequality implies

$$
\begin{aligned}
E\left(N_{s}-N_{t}\right)^{k} & \leq 2\left(\frac{1}{s}-\frac{1}{t}\right) s^{-k} E N_{\varepsilon}^{k} \\
& \leq 2\left(\frac{m}{\varepsilon}\right)^{m} E N_{\varepsilon}^{m}\left(\frac{1}{s}-\frac{1}{t}\right)=\widetilde{c}_{m}(\varepsilon)\left(\frac{1}{s}-\frac{1}{t}\right),
\end{aligned}
$$

where $\widetilde{c}_{m}(\varepsilon)$ is finite since $E N_{\varepsilon}^{m}<\infty$ [see van Zwet (1978)]. For $t \geq 1 / m$, $1 / s-1 / t \leq 1 / \varepsilon t \leq m / \varepsilon$, and this yields (4.2). Insertion of this into (4.1) suffices to cover the case of $t<1 / m$ so that

$$
\left|\kappa_{m}\left(N_{s}-N_{t}\right)\right| \leq c_{m}^{\prime}(\varepsilon)(1 / s-1 / t) \quad \text { for } \varepsilon t \leq s<t<1 .
$$

But this yields (4.3) for some $c_{m}(\varepsilon)>c_{m}^{\prime}(\varepsilon)$ and the proof is complete.

Recall that $N_{t}(x)$ denotes the number of points among $X_{1}, \ldots, X_{N_{t}}$ which fall in $(0, x], 0 \leq x \leq 1$. Suppose that among the $N_{t}(x)$ points in $(0, x], \xi$ is the first point in $[x-t, x]$ and that we delete all points in $(\xi, x]$ that follow it. Let $N_{t / x}^{\prime}$ denote the number of points remaining. Clearly $N_{t}(x) \geq N_{t / x}^{\prime}$ and $N_{t / x}^{\prime}$ is distributed like $N_{t / x}$. Note that for fixed $x$ the processes $\left\{N_{t / x}^{\prime}: 0<t<x\right\}$ and $\left\{N_{t / x}: 0<t<x\right\}$ also have the same distribution.

Furthermore, $\left(N_{t}(x)-N_{t / x}^{\prime}\right)$ is stochastically smaller than $N_{1 / 2}-2$. To see this, note that among the points $X_{1}, \ldots, X_{N_{t}}$, the first points $\xi$ and $\xi^{\prime}$ in $[x-t, x]$ and $(x, x+t]$, respectively, plus all points in $\left(\xi, \xi^{\prime}\right)$ form a Kakutani splitting of the 
interval $(x-t, x+t)$ into intervals of length $\leq t$, which is half the width of the interval.

Summarizing, we have a process $N_{t / x}^{\prime}$ distributed like $N_{t / x}$ and such that

$$
N_{t}(x)=N_{t / x}^{\prime}+R(x, t)
$$

where, for every $x$ and $t$,

$$
0 \leq R(x, t) \stackrel{\text { st }}{\leq} N_{1 / 2}-2
$$

LEMMA 4.4. For every $\varepsilon>0$ and $m=2,3, \ldots$, there exists a positive number $\widetilde{c}_{m}(\varepsilon)$ such that

$$
\left|\mu_{m}\left(N_{s}(x)-N_{t}(x)\right)\right| \leq \widetilde{c}_{m}(\varepsilon)\left\{\left(\frac{x}{s}-\frac{x}{t}\right)^{m / 2}+1\right\}
$$

for $\varepsilon t \leq s<t<x \leq 1$.

PROOF. In view of (4.4) and (4.3),

$$
\begin{aligned}
& \left|\mu_{m}\left(N_{s}(x)-N_{t}(x)\right)\right| \\
& =\left|\mu_{m}\left(N_{s / x}^{\prime}-N_{t / x}^{\prime}+R(x, s)-R(x, t)\right)\right| \\
& =\left|\sum_{k=0}^{m}\left(\begin{array}{c}
m \\
k
\end{array}\right) \mu_{k}(R(x, s)-R(x, t)) \mu_{m-k}\left(N_{s / x}^{\prime}-N_{t / x}^{\prime}\right)\right| \\
& \leq c_{m}(\varepsilon)\left\{\left(\frac{x}{s}-\frac{x}{t}\right)^{m / 2}+\left(\frac{x}{s}-\frac{x}{t}\right)\right\}+\left|\mu_{m}(R(x, s)-R(x, t))\right| \\
& \quad+\sum_{k=2}^{m-2}\left(\begin{array}{c}
m \\
k
\end{array}\right)\left|\mu_{k}(R(x, s)-R(x, t))\right| c_{m-k}(\varepsilon) \\
& \quad \times\left\{\left(\frac{x}{s}-\frac{x}{t}\right)^{(m-k) / 2}+\left(\frac{x}{s}-\frac{x}{t}\right)\right\} .
\end{aligned}
$$

Now (4.5) implies that

$$
\left|\mu_{k}(R(x, s)-R(x, t))\right| \leq 2^{2 k-1}\left\{E|R(x, s)|^{k}+E|R(x, t)|^{k}\right\} \leq 2^{k} E\left(N_{1 / 2}-2\right)^{k}
$$

and since $N_{1 / 2}$ has finite moments of every order, the proof is complete.

Lemma 4.5. For every $0<a<A$,

$$
\lim _{n \rightarrow \infty} \sup _{\substack{0 \leq x \leq 1 \\ a n^{1 / 2} \leq\left|\tau^{-1}-n / 2\right| \leq A n^{1 / 2}}}\left|\frac{N_{2 / n}(x)-N_{\tau}(x)}{n-2 / \tau}-x\right|=0 \quad \text { a.s. }
$$


Proof. Fix $x \in(0,1]$. Consider two sequences $s_{n}$ and $t_{n}$ such that $\varepsilon \max \left(s_{n}, t_{n}\right) \leq \min \left(s_{n}, t_{n}\right)<\max \left(s_{n}, t_{n}\right)<x \leq 1$ and $n^{-\delta}\left|\frac{1}{s_{n}}-\frac{1}{t_{n}}\right| \rightarrow \infty$ for some positive $\varepsilon$ and $\delta$. Then (4.6) implies that for every $m=1,2, \ldots$,

$$
P\left(\left|N_{S_{n}}(x)-N_{t_{n}}(x)-E N_{S_{n}}(x)+E N_{t_{n}}(x)\right| \geq n^{-\delta / 4}\left|\frac{x}{s_{n}}-\frac{x}{t_{n}}\right|\right)=o\left(n^{-\delta m / 4}\right)
$$

as $n \rightarrow \infty$. Hence, by choosing $m>4 / \delta$,

$$
\lim _{n} \frac{\left|N_{s_{n}}(x)-N_{t_{n}}(x)-E N_{s_{n}}(x)+E N_{t_{n}}(x)\right|}{\left|x / s_{n}-x / t_{n}\right|}=0 \quad \text { a.s. }
$$

For $s, t<x,(4.4),(4.5)$ and (1.6) insure that $\left|E\left(N_{s}(x)-N_{t}(x)\right)-2 x / s+2 x / t\right| \leq$ $E\left(N_{1 / 2}-2\right)=1$ and hence

$$
\lim _{n \rightarrow \infty} \frac{N_{s_{n}}(x)-N_{t_{n}}(x)}{2 x / s_{n}-2 x / t_{n}}=1 \quad \text { a.s. }
$$

for every pair of sequences satisfying the above requirements.

Take $s_{n}=2 / n$ and define $t_{n, k}^{-1}=n / 2+k \eta n^{1 / 2}$ where

$$
k \in \mathcal{g}=\left\{ \pm\left[\frac{a}{\eta}\right], \pm\left(\left[\frac{a}{\eta}\right]+1\right), \pm\left(\left[\frac{a}{\eta}\right]+2\right), \ldots, \pm\left(\left[\frac{A}{\eta}\right]+1\right)\right\}
$$

and $\eta$ is a fixed (small) positive number. For each $k \in \mathcal{g}, s_{n}=2 / n$ and $t_{n, k}$ satisfy the requirements for (4.7), so

$$
\lim _{n \rightarrow \infty} \max _{k \in \mathscr{g}}\left|\frac{N_{2 / n}(x)-N_{t_{n, k}}(x)}{n-2 / t_{n, k}}-x\right|=0 \quad \text { a.s. }
$$

Let $\tau_{n}$ be a sequence with $a n^{1 / 2} \leq\left|\tau_{n}^{-1}-n / 2\right| \leq A n^{1 / 2}$. Then there exist $k_{n}, k_{n}+1 \in \mathcal{g}$ such that $t_{n, k_{n}}^{-1} \leq \tau_{n}^{-1} \leq t_{n, k_{n}+1}^{-1}$. Now $t_{n, k_{n}+1}^{-1}-t_{n, k_{n}}^{-1}=\eta n^{1 / 2}$ and $\left|t_{n, k_{n}}^{-1}-n / 2\right| \geq[a / \eta] \eta n^{1 / 2}$, so for $\eta \leq a / 2$,

$$
\begin{aligned}
\limsup _{n} & \left|\frac{N_{\tau_{n}}(x)-N_{t_{n, k_{n}}}(x)}{n-2 / t_{n, k_{n}}}\right| \\
& \leq \limsup _{n}\left|\frac{N_{t_{n, k_{n}+1}}(x)-N_{t_{n, k}}(x)}{2 / t_{n, k_{n}+1}-2 / t_{n, k_{n}}}\right| \frac{1}{[a / \eta]} \\
& \leq \frac{2 \eta}{a} \limsup _{n} \max _{k \in \mathcal{g}}\left|\frac{N_{t_{n, k+1}}(x)-N_{t_{n, k}}(x)}{2 / t_{n, k+1}-2 / t_{n, k}}\right| \\
& \leq \frac{2 \eta x}{a} \leq \frac{2 \eta}{a} \quad \text { a.s., }
\end{aligned}
$$

since the pair of sequences $t_{n, k}$ and $t_{n, k+1}$ satisfy the requirements for (4.7). Also

$$
\limsup _{n}\left|\frac{n-2 / \tau_{n}}{n-2 / t_{n, k_{n}}}-1\right| \leq \frac{1}{[a / \eta]} \leq \frac{2 \eta}{a} .
$$


Combining (4.8)-(4.10) and noting that $\eta>0$ may be taken arbitrarily small, we find that for fixed $x \in(0,1]$,

(4.11) $\lim _{n \rightarrow \infty} \sup _{a n^{1 / 2} \leq\left|\tau_{n}^{-1}-n / 2\right| \leq A n^{1 / 2}}\left|\frac{N_{2 / n}(x)-N_{\tau_{n}}(x)}{n-2 / \tau_{n}}-x\right|=0 \quad$ a.s.

Since $\left(N_{2 / n}(x)-N_{\tau_{n}}(x)\right) /\left(n-2 / \tau_{n}\right)$ is nondecreasing in $x$ and equals 0 for $x=0$, a standard argument completes the proof.

Let $n(x)=\sum_{i=1}^{n} \mathbb{1}_{(0, x]}\left(X_{i}\right)$ be the number of points among $X_{1}, \ldots, X_{n}$ that fall in $(0, x]$, so that $F_{n}(x)=\frac{n(x)}{n}$ is the empirical d.f. of $X_{1}, \ldots, X_{n}$.

LEMMA 4.6. As $n \rightarrow \infty$,

$$
\sup _{0 \leq x \leq 1} n^{1 / 2}\left|F_{N_{2 / n}}(x)-F_{n}(x)\right| \rightarrow 0
$$

in probability.

ProOF. We have

$$
n^{1 / 2}\left|F_{N_{2 / n}}(x)-F_{n}(x)\right|=\frac{n^{1 / 2}\left|N_{2 / n}-n\right|}{N_{2 / n}}\left|\frac{N_{2 / n}(x)-n(x)}{N_{2 / n}-n}-F_{n}(x)\right| .
$$

By (1.3), (1.6), (1.7) and (1.11), it suffices to show that

$$
n^{-1 / 2}\left|N_{2 / n}-n\right| \sup _{0 \leq x \leq 1}\left|\frac{N_{2 / n}(x)-n(x)}{N_{2 / n}-n}-x\right| \stackrel{P}{\rightarrow} 0 .
$$

The definition of $M_{n}$ following (1.1) implies that $N_{M_{n}}=n$, so $N_{M_{n}}(x)=n(x)$. Applying Lemma 4.5 twice, once for general $x$ and once for $x=1$ and substituting $\tau=M_{n}$, we find that, for every $0<a<A$,

$$
\lim _{n \rightarrow \infty} \sup _{0 \leq x \leq 1}\left|\frac{N_{2 / n}(x)-n(x)}{N_{2 / n}-n}-x\right| \mathbb{1}_{[a, A]}\left(n^{-1 / 2}\left|M_{n}^{-1}-n / 2\right|\right)=0 \quad \text { a.s. }
$$

and since $n^{-1 / 2}\left|N_{2 / n}-n\right|$ is bounded in probability by (1.6) and (1.7), we have, for every $0<a<A$,

$$
\begin{gathered}
n^{-1 / 2}\left|N_{2 / n}-n\right| \sup _{0 \leq x \leq 1}\left|\frac{N_{2 / n}(x)-n(x)}{N_{2 / n}-n}-x\right| \\
\times \mathbb{1}_{[a, A]}\left(n^{-1 / 2}\left|M_{n}^{-1}-n / 2\right|\right) \rightarrow 0 \quad \text { a.s. }
\end{gathered}
$$

We have $\left\{M_{n}>t\right\}=\left\{N_{t}>n\right\}$ and hence, if $n-2 / t+1>0$ and $0<t<1$,

$$
P\left(M_{n}>t\right)=P\left(\frac{N_{t}-E N_{t}}{\sigma\left(N_{t}\right)}>\frac{n-2 / t+1}{c^{1 / 2}} t^{1 / 2}\right)
$$

$$
\leq \frac{c}{t(n-2 / t+1)^{2}} \text {, }
$$


and therefore, for sufficiently large $n$,

$$
\begin{aligned}
& P\left(n^{-1 / 2}\left(M_{n}^{-1}-n / 2\right)<-A\right) \\
& \quad=P\left(M_{n}>\left(n / 2-A n^{1 / 2}\right)^{-1}\right) \leq \frac{c\left(n / 2-A n^{1 / 2}\right)}{\left(2 A n^{1 / 2}+1\right)^{2}} \leq \frac{c}{8 A^{2}} .
\end{aligned}
$$

This probability can be made arbitrarily small by taking $A$ large and the same is true for $P\left(n^{-1 / 2}\left(M_{n}^{-1}-n / 2\right)>A\right)$, so (4.13) can be extended to

$$
\begin{gathered}
n^{-1 / 2}\left|N_{2 / n}-n\right| \sup _{0 \leq x \leq 1}\left|\frac{N_{2 / n}(x)-n(x)}{N_{2 / n}-n}-x\right| \\
\times \mathbb{1}_{[a, \infty)}\left(n^{-1 / 2}\left|M_{n}^{-1}-n / 2\right|\right) \stackrel{P}{\rightarrow} 0 .
\end{gathered}
$$

Finally we consider the set $B=\left\{n^{-1 / 2}\left|M_{n}^{-1}-n / 2\right| \leq a\right\}$. Writing $s_{n}^{-1}=$ $\left(n / 2+a n^{1 / 2}\right)$ and $t_{n}^{-1}=\left(n / 2-a n^{1 / 2}\right)$ we see that on the set $B, N_{t_{n}} \leq n \leq N_{s_{n}}$ and since $s_{n}<2 / n<t_{n}$, we have $\left|N_{2 / n}-n\right| \leq\left|N_{s_{n}}-N_{t_{n}}\right|$ on $B$. Hence, by Lemma 4.3, we have for sufficiently large $n$ and any $\delta, \varepsilon>0$,

$$
\begin{gathered}
P\left(n^{-1 / 2}\left|N_{2 / n}-n\right| \sup _{0 \leq x \leq 1}\left|\frac{N_{2 / n}(x)-n(x)}{N_{2 / n}-n}-x\right| \mathbb{1}_{B} \geq \delta\right) \\
\leq P\left(n^{-1 / 2}\left|N_{s_{n}}-N_{t_{n}}\right| \geq \delta\right) \leq \frac{E\left(N_{s_{n}}-N_{t_{n}}\right)^{2}}{\delta^{2} n} \\
\quad \leq \frac{2 c_{2}(1 / 2) a n^{1 / 2}+16 a^{2} n}{\delta^{2} n} \leq 17 \frac{a^{2}}{\delta^{2}} \leq \varepsilon,
\end{gathered}
$$

if we take $a$ sufficiently small. Together (4.15) and (4.16) imply (4.12) and the lemma.

To complete the proof of Theorem 4.1, it suffices now to take $s=2 / n$ in $U(\cdot, s)$ to see that

$$
\left\{(n / 2)^{1 / 2}\left(F_{N_{2 / n}}(x)-x\right) ; 0<x<1\right\} \stackrel{L}{\rightarrow} \sigma B_{0}
$$

by Theorem 3.1. It then follows from Lemma 4.6 that the proof is complete.

5. Weak convergence of the $W(\cdot, t)$ and $V(\cdot, t)$ processes. We now prove that the stopped empirical processes of the relative spacings, $V(\cdot, t)$, converge weakly on $[0,1]$ to a Gaussian process $V(\cdot)$ as $t \rightarrow 0$. The proof concentrates in fact upon establishing the weak convergence on $[0,1]$ of the related processes $W(\cdot, s)$ of which the $V$-processes are tied-down versions; see (1.17), (1.18) and (1.20). The proof is based on a representation of $W(\cdot, t)$ as a sum of independent processes. As in Section 3, let $\widetilde{F}_{s}=\sigma\left(D_{s i}: 1 \leq i \leq N_{s}+1\right)$ be the $\sigma$-field of the partitions at level $s$. For any $0<t<s<1$, we may write

$$
K(x, t)=\sum_{i=1}^{N_{s}+1} K^{(i)}\left(x / D_{s i}, t / D_{s i}\right)
$$


where $K^{(i)}, 1 \leq i \leq N_{s}+1$, are independent copies of $K$ that are independent also of $\mathcal{F}_{s}$. From this and the definition of $W(\cdot, t)$ in $(1.18)$, we get the following key representation of $W(\cdot, t)$ as a sum of conditionally independent processes, namely, for any $0<t<s<1$,

$$
W(\cdot, t)=\sum_{i=1}^{N_{s}+1} D_{s i}^{1 / 2} W^{(i)}\left(\cdot, t / D_{s i}\right)
$$

in which $W^{(i)}, 1 \leq i \leq N_{s}+1$, are independent copies of $W$ that are also independent of $\mathcal{F}_{s}$. The visual simplicity of this representation is due to the definition (1.18) in which the centering for $(r / 2) K(y r, r)$ is chosen to be $y$ rather than its mean when $y>1$ or $r \geq 1$. Since we are only concerned with the processes $W(y, t)$ for $0 \leq y \leq 1$, the case of $y>1$ plays no role in (5.2), but since $t / D_{s i}$ may exceed 1 , the case of $r \geq 1$ does. When only terms centered at expectations are used, the expression (5.2) becomes

$$
\begin{aligned}
W(y, t)= & \sum_{i: D_{s i}>t} D_{s i}^{1 / 2} W^{(i)}\left(y, t / D_{s i}\right) \\
& +(t / s)^{1 / 2} W(y t / s, s)+2 y(s / t)^{1 / 2} \int_{t / s}^{1} z W(d z, s),
\end{aligned}
$$

for $0 \leq y \leq 1$ and $0<t<s<1$. It is in this form that the recursion is used in Section 6 . Note that the full integral over $[0,1]$ in the above is zero.

We first use the representation (5.2) to prove the limiting normality of the finite-dimensional distributions. We do this by applying the Lindeberg central limit theorem to the sum in (5.2) conditionally given $\mathcal{F}_{s}$. [The remark following (3.10) should be noted for this section as well.] Since we need to compute moments using Theorem 2.2, we split the summation of (5.2) into two parts according as $t / D_{s i}>$ $1 / 4$ or $\leq 1 / 4$. Write $W(\cdot, t)=W_{s}^{-}(\cdot, t)+W_{s}^{+}(\cdot, t)$, where $W_{s}^{-}$represents the summation over those $i$ for which $D_{s i}<4 t$. Recall from (1.8) that for $0 \leq y \leq 1$ and $0<u<1, E W(y, u)=0$. Then, conditionally given $\mathcal{F}_{s}, W_{s}^{-}$and $W_{s}^{+}$are independent and $W_{s}^{+}$has mean zero, so that for $0 \leq y \leq 1$ and $0<t<s<1$,

$$
\operatorname{var}_{s}\{W(y, t)\}=\operatorname{var}_{s}\left\{W_{s}^{-}(y, t)\right\}+E_{s}\left[W_{s}^{+}(y, t)\right]^{2},
$$

where $\operatorname{var}_{s}, E_{s}, P_{s}$ indicate the conditional quantities given $\mathcal{F}_{s}$. Also, for $0<t \leq 1 / 2$ and $m=2$, Corollary 2.3 implies

$$
E_{S}\left[W_{s}^{+}(y, t)\right]^{2}=\sum_{i: D_{s i} \geq 4 t} D_{s i} E_{s}\left[W\left(y, t / D_{s i}\right)\right]^{2}=\frac{1}{2} c_{2, y} \sum^{+} D_{s i} \leq \frac{1}{2} c_{2, y}
$$

where $c_{2, y}$ is the constant of Corollary 2.3 for $m=2$ and $\alpha=y$, and $\sum^{+}$denotes summation over $\left\{i: D_{s i} \geq 4 t\right\}$. [The actual covariance function for the $W(\cdot, t)$ processes, and hence for the limiting $W(\cdot)$ process, is derived in (7.10) where in particular, $c_{2, y}=\alpha(y, y)$ with $\alpha$ defined in (7.9).] 
Next we deal with $W^{-}(y, t)$. Since $E_{s}\left[W\left(y, t / D_{s i}\right)\right]=0$ whenever $D_{s i}>t$, we find in view of (1.8) and (1.14) that with probability 1 ,

$$
\begin{aligned}
\left|E_{S}\left[W_{s}^{-}(y, t)\right]\right| & =\left|\sum_{i: D_{s i} \leq t} D_{s i}^{1 / 2} E_{S} W\left(y, t / D_{s i}\right)\right| \\
& =(t / 2)^{1 / 2}\left|\sum_{i: D_{s i} \leq t}\left[E_{S} K\left(y t / D_{s i}, t / D_{s i}\right)-(2 y / t) D_{s i}\right]\right| \\
& \leq(t / 2)^{1 / 2} \sum_{i: D_{s i} \leq y t} 1+y(2 / t)^{1 / 2} \sum_{i: D_{s i} \leq t} D_{s i} \\
& \leq(t / 2)^{1 / 2} K(y t, s)+y(2 / t)^{1 / 2} t K(t, s)=O\left(t^{3 / 2} / s^{2}\right) .
\end{aligned}
$$

To handle the variance of $W^{-}(y, t)$, observe first that $W(y, u)$ is nonrandom when $u \geq 1$. Thus

$$
\begin{aligned}
\operatorname{var}_{s}\left\{W^{-}(y, t)\right\} & =\sum_{i: D_{s i}<4 t} D_{s i} \operatorname{var}_{s}\left\{W\left(y, t / D_{s i}\right)\right\} \\
& \leq \sum_{t<D_{s i}<4 t} D_{s i} E_{s}\left[W\left(y, t / D_{s i}\right)\right]^{2} \\
& \leq 4 t K(4 t, s) \sup _{1 / 4<u<1} E[W(y, u)]^{2} .
\end{aligned}
$$

By (1.14), $t K(4 t, s) \rightarrow 0$ a.s. as $s \rightarrow 0$ if $t=t(s)$ is chosen so that $t / s \rightarrow 0$. Moreover, the quantity $C(y):=\sup _{1 / 4<u<1} E[W(y, u)]^{2}$ is finite, since, for $1 / 4 \leq$ $u<1$,

$$
E[W(y, u)]^{2} \leq E\left[\frac{u}{2} K^{2}(u y, u)\right] \leq \frac{u}{2} E\left(N_{u}+1\right)^{2} \leq \frac{1}{2} E\left(N_{1 / 4}+1\right)^{2}
$$

which is finite; see van Zwet (1978). Thus

$$
W_{s}^{-}(y, t) \stackrel{P}{\rightarrow} 0 \quad \text { as } s \rightarrow 0 \text { and } t^{3} / s^{4} \rightarrow 0 .
$$

Consider now the limiting finite-dimensional distributions of $W_{s}^{+}(\cdot, t)$. The Lindeberg criterion (conditional given $\mathcal{F}_{S}$ ) for the one-dimensional case involves

$$
L_{t}(\varepsilon):=\sum^{+} D_{s i} E_{s}\left\{\left[W^{(i)}\left(y, t / D_{s i}\right)\right]^{2} \mathbb{1}_{\left[D_{s i}^{1 / 2}\left|W^{(i)}\left(y, t / D_{s i}\right)\right| \geq \varepsilon B_{t}^{1 / 2}\right]}\right\}
$$

for $\varepsilon>0$ and $B_{t}=\operatorname{var}_{s}\left(W_{s}^{+}(y, t)\right)$. From Hölder's and Chebyshev's inequalities we obtain

$$
\begin{aligned}
L_{t}(\varepsilon) & \leq \sum^{+} D_{s i}\left\{E_{S}\left[W^{(i)}\left(y, t / D_{s i}\right)\right]^{4} P_{s}\left[D_{s i}^{1 / 2}\left|W^{(i)}\left(y, t / D_{s i}\right)\right| \geq \varepsilon B_{t}^{1 / 2}\right]\right\}^{1 / 2} \\
& \leq \sum^{+} D_{s i}^{3 / 2}\left\{E_{s}\left[W^{(i)}\left(y, t / D_{s i}\right)\right]^{4} E_{s}\left[W^{(i)}\left(y, t / D_{s i}\right)\right]^{2}\right\}^{1 / 2} / \varepsilon B_{t}^{1 / 2} .
\end{aligned}
$$


Since $t / D_{s i} \leq 1 / 4$, the moments in the summation are bounded by constants (for fixed $y$ ) by Corollary 2.3. Also, $B_{t}$ converges to the nonzero constant $c_{2, y} / 2$ by (5.4) and the sentence following (5.6). Since each $D_{s i} \leq s$ by definition, it follows from the above that $L_{t}(\varepsilon) / B_{t}=O\left(s^{1 / 2}\right)=o(1)$ as $s \rightarrow 0$ provided only that $t \rightarrow 0$ as well. Thus Lindeberg's condition is satisfied, and therefore the one-dimensional distributions of $W(\cdot, t)$ converge to those of $W(\cdot)$. For higher dimensions, the proof is similar requiring only that finite linear combinations $\sum a_{j} W\left(y_{j}, t\right)$ be considered. We have therefore established the following lemma.

LEMma 5.1. As $t \rightarrow 0$, the finite-dimensional distributions of $\{W(y, t): 0 \leq$ $y \leq 1\}$ converge to those of $W(\cdot)$, a mean zero Gaussian process on $[0,1]$ with covariance given in (7.9) and (7.10).

To complete the proof of weak convergence, we will apply a standard sufficient condition for tightness in $D[0,1]$ that is based on a moment bound for adjacent increments of the process; see Theorem 15.6 of Billingsley (1968). The appropriate bound is given in the following lemma. (We gratefully acknowledge our appreciation to Christian Genest for pointing out an error in an earlier attempt to prove this result based only on a moment bound for a single interval.)

Write $J_{1}=(x, y], J_{2}=(y, z]$ for $0 \leq x<y<z \leq 1$, and recall that we write, for example, $W\left(J_{1}, t\right)=W(y, t)-W(x, t)$. The adjacency and interval structure of $J_{1}$ and $J_{2}$ is not required in the moment bound that we now derive, and so we state it for general disjoint Borel sets. Observe that although $K(\cdot, t)$ and $W(\cdot, t)$ are defined as point functions, since they are clearly equivalent to (signed) measures, this enables us to write $K(B, t)$ and $W(B, t)$ unambiguously for any Borel set $B$ as well as for intervals.

LEMMA 5.2. There exists a constant $C$ such that for all $t \in(0,1]$ and any disjoint Borel subsets $J_{1}$ and $J_{2}$ of $[0,1]$,

$$
E\left[W\left(J_{1}, t\right) W\left(J_{2}, t\right)\right]^{2} \leq C\left|J_{1}\right|\left|J_{2}\right| .
$$

PROOF. Assume first of all that $0<t \leq 1 / 4$. [The reader should note that for its application to tightness, the bound of (5.7) is only needed for $t$ in some interval of the form $\left(0, t_{0}\right)$.] By applying Theorem 2.4 to the pair $D_{i}(t)=K\left(t J_{i}, t\right)$, $i=1,2$, we get for $j, k=0,1,2$, that

$$
\kappa_{j, k}(t) \equiv \kappa_{j, k}\left(D_{1}(t), D_{2}(t)\right)=\frac{c_{j, k}}{t} \quad \text { for } 0<t \leq \frac{1}{4} .
$$

This joint cumulant may be expressed in terms of central moments; specifically, 
a straightforward computation shows that if $E X=\mu, E Y=v$,

$$
\begin{aligned}
& \kappa_{2,0}(X, Y)=\sigma_{X}^{2}, \\
& \kappa_{0,2}(X, Y)=\sigma_{Y}^{2}, \\
& \kappa_{1,1}(X, Y)=\operatorname{Cov}(X, Y) \text { and } \\
& \kappa_{2,2}(X, Y)=E(X-\mu)^{2}(Y-\nu)^{2}-\sigma_{X}^{2} \sigma_{Y}^{2}-2[\operatorname{Cov}(X, Y)]^{2} .
\end{aligned}
$$

Thus, using $W\left(J_{1}, t\right)=X$ and $W\left(J_{2}, t\right)=Y$, this means by (1.8) and the scalar homogeneity evident in (5.9), that for $0<t \leq 1 / 4$,

$$
\begin{aligned}
E\left[W\left(J_{1}, t\right) W\left(J_{2}, t\right)\right]^{2}= & \left(\frac{t}{2}\right)^{2} \kappa_{2,2}(t)+E\left[W\left(J_{1}, t\right)\right]^{2} E\left[W\left(J_{2}, t\right)\right]^{2} \\
& +2\left\{E W\left(J_{1}, t\right) W\left(J_{2}, t\right)\right\}^{2} \\
= & \left(\frac{t}{2}\right)^{2}\left\{\kappa_{2,2}(t)+\kappa_{2,0}(t) \kappa_{0,2}(t)+2\left[\kappa_{1,1}(t)\right]^{2}\right\} \\
= & \frac{t}{4} c_{2,2}+\frac{1}{4} c_{2,0} c_{0,2}+\frac{1}{2} c_{1,1}^{2},
\end{aligned}
$$

with the last equation following from (5.8). It remains, then, to obtain bounds for the constants $c_{22}, c_{20}$ and $c_{11}$ in terms of $\left|J_{1}\right|$ and $\left|J_{2}\right|$ so as to verify (5.7) when $0<t \leq 1 / 4$.

By definition, and in view of (5.9) and (1.21), it follows that for any $0<t \leq 1 / 4$,

$$
\begin{aligned}
c_{2,2}= & t \kappa_{2,2}(t)=t \kappa_{2,2}\left(D_{1}(t), D_{2}(t)\right) \\
& \leq t E\left(\left\{D_{1}(t)-E D_{1}(t)\right\}^{2}\left\{D_{2}(t)-E D_{2}(t)\right\}^{2}\right) \\
& =t E\left(\left\{D_{1}(t)-2 t^{-1}\left|J_{1}\right|\right\}^{2}\left\{D_{2}(t)-2 t^{-1}\left|J_{2}\right|\right\}^{2}\right) \\
& \leq t\left(E\left\{D_{1}(t) D_{2}(t)\right\}^{2}+\left(2 t^{-1}\left|J_{1}\right|\right)^{2} E\left[D_{2}(t)\right]^{2}+\left(2 t^{-1}\left|J_{2}\right|\right)^{2} E\left[D_{1}(t)\right]^{2}\right. \\
& \left.\quad+16 t^{-2}\left|J_{1}\right|\left|J_{2}\right| E\left(D_{1}(t) D_{2}(t)\right)+16 t^{-4}\left|J_{1}\right|^{2}\left|J_{2}\right|^{2}\right) \\
& \leq t E\left\{D_{1}(t) D_{2}(t)\right\}^{2}+C_{t}\left\{\left|J_{1}\right| E\left[D_{2}(t)\right]^{2}+\left|J_{2}\right| E\left[D_{1}(t)\right]^{2}+\left|J_{1}\right|\left|J_{2}\right|\right\}
\end{aligned}
$$

for some constant $C_{t}$ depending on the chosen t but not on the $J_{i}$ 's. For this, recall that $D_{i}(t) \leq\left(N_{t}+1\right)$ and $N_{t}$ has finite moments by Lemma 2.1. Thus to obtain the desired bound for $c_{2,2}$, it suffices to bound $E\left\{D_{1}(t) D_{2}(t)\right\}^{2}$ and $E\left[D_{i}(t)\right]^{2}$ appropriately for some value of $t \leq 1 / 4$. To bound the other two terms of (5.10) observe first that by Cauchy-Schwarz, $c_{1,1}^{2} \leq c_{2,0} c_{0,2}$. Hence it suffices to establish an appropriate bound for the right-hand side of

$$
c_{2,0}=t E\left\{K\left(t J_{1}, t\right)-\frac{2}{t}\left|J_{1}\right|\right\}^{2} \leq t E\left[K\left(t J_{1}, t\right)\right]^{2}
$$

for some $0<t \leq 1 / 4$. Such bounds are contained in the following: 
LEMMA 5.3. For any Borel set $J \subset[0,1], 0<t<1$ and $k=1,2, \ldots$,

$$
E[K(t J, t)]^{k} \leq 2^{k}|J| E N_{t}^{k},
$$

and for any disjoint Borel sets $J_{1}$ and $J_{2}$ in $[0,1]$ and $0<t<1$,

$$
E\left[K\left(t J_{1}, t\right) K\left(t J_{2}, t\right)\right]^{2} \leq C\left|J_{1}\right|\left|J_{2}\right| E N_{t}^{4}
$$

for some constant $C$.

PROOF. The first inequalities are the simplest to prove since they involve only one set $J$. (Although only the case $k=2$ is needed here, we give it for general $k$ since this requires no new ideas.) For this single $J$, write $D(t)=K(t J, t)$. We use the representation

$$
0 \leq D(t)=\sum_{j=1}^{N_{t}} \eta_{j}
$$

where the r.v. $\eta_{j}$ equals the number of new spacings with length in $t J$ that originate with the $j$ th splitting, which occurs at $X_{j}$. Since $\eta_{j}=0,1$ or 2 , we have for any $k \geq 1$ that

$$
\begin{aligned}
E[D(t)]^{k} & =E\left[\sum_{j=1}^{N_{t}} \eta_{j}\right]^{k}=\sum_{j_{1}=1}^{\infty} \cdots \sum_{j_{k}=1}^{\infty} E\left(\prod_{i=1}^{k} \eta_{j_{i}} \mathbb{1}_{\left[N_{t} \geq \max _{i} j_{i}\right]}\right) \\
& \leq 2^{k-1} \sum_{j_{1}=1}^{\infty} \cdots \sum_{j_{k}=1}^{\infty} E\left(\eta_{\max j_{i}} \mathbb{1}_{\left[N_{t} \geq \max j_{i}\right]}\right) \\
& =2^{k-1} \sum_{j=1}^{\infty}\left(j^{k}-(j-1)^{k}\right) E\left(\eta_{j} \mathbb{1}_{\left[N_{t} \geq j\right]}\right) \\
& =2^{k-1} \sum_{j=1}^{\infty}\left(j^{k}-(j-1)^{k}\right) P\left[N_{t} \geq j\right] E\left[\eta_{j} \mid N_{t} \geq j\right] .
\end{aligned}
$$

On the event $\left[N_{t} \geq j\right], X_{j}$ splits an interval whose length exceeds $t$. Hence, since the splitting is done uniformly, $E\left[\eta_{j} \mid N_{t} \geq j\right] \leq 2|J|$ and so, for any $0<t \leq 1$,

$$
E[D(t)]^{k} \leq 2^{k}|J| \sum_{j=1}^{\infty} j^{k} P\left[N_{t}=j\right]=2^{k}|J| E N_{t}^{k} .
$$

To prove the second inequality (5.13), let $\eta_{i j}$ equal the number of the two new spacings that originate with the $i$ th splitting, whose lengths are in $t J_{j}$. Thus, each $\eta_{i j} \in\{0,1,2\}$, and

$$
D_{j}(t)=K\left(t J_{j}, t\right)=\sum_{i=1}^{N_{t}} \eta_{i j}=\sum_{i=1}^{\infty} \eta_{i j} \mathbb{1}_{\left[N_{t} \geq i\right]} .
$$


Recall that $M_{n}$ is the maximum spacing after $n$ splittings. Write

$$
\begin{aligned}
& E K^{2}\left(t J_{1}, t\right) K^{2}\left(t J_{2}, t\right) \\
& =E \sum_{i=1}^{\infty} \sum_{k=1}^{\infty} \sum_{j=1}^{\infty} \sum_{l=1}^{\infty} \eta_{i 1} \eta_{k 1} \eta_{j 2} \eta_{l 2} \mathbb{1}_{\left[N_{t} \geq \max \{i, j, k, l\}\right]} \\
& \leq 4 \sum_{n=1}^{\infty} \sum_{\substack{i \leq n \\
i \vee j=n}} \sum_{j \leq n}\left(i^{2}-(i-1)^{2}\right)\left(j^{2}-(j-1)^{2}\right) E\left(\eta_{i 1} \eta_{j 2} \mathbb{1}_{\left[N_{t} \geq n\right]}\right) .
\end{aligned}
$$

Set

$$
\begin{aligned}
A_{i} & =\frac{t J_{1}}{M_{i-1}} \cup\left(1-\frac{t J_{1}}{M_{i-1}}\right) \text { and } \\
B_{j} & =\frac{t J_{2}}{M_{j-1}} \cup\left(1-\frac{t J_{2}}{M_{j-1}}\right),
\end{aligned}
$$

where for $J \subset \mathbb{R}$ and $a, b \in \mathbb{R}, a-b J=\{a-b x: x \in J\}$. Note that the measures of $A_{i}$ and $B_{j}$ satisfy

$$
\left|A_{i}\right| \leq \frac{2 t}{M_{i-1}}\left|J_{1}\right|, \quad\left|B_{j}\right| \leq \frac{2 t}{M_{j-1}}\left|J_{2}\right|
$$

for all $i$ and $j$. By definition,

$$
\begin{aligned}
\eta_{m 1} & =\mathbb{1}_{\left[\left(1-U_{m}\right) M_{m-1} \in t J_{1}\right]}+\mathbb{1}_{\left[U_{m} M_{m-1} \in t J_{1}\right]} \\
& \leq 2 \mathbb{1}_{\left[U_{m} \in\left(t J_{1} / M_{m-1}\right) \cup\left(1-t J_{1} / M_{m-1}\right)\right]}=2 \mathbb{1}_{\left[U_{m} \in A_{m}\right]},
\end{aligned}
$$

with a similar bound holding for $\eta_{m 2}$. Substitution of this into (5.15) yields

$$
\begin{aligned}
& E K^{2}\left(t J_{1}, t\right) K^{2}\left(t J_{2}, t\right) \\
& \quad \leq 64 \sum_{n=1}^{\infty} \sum_{\substack{i \leq n \\
i \vee j=n}} \sum_{\substack{j \leq n \\
j}} i j\left[U_{i} \in A_{i}, U_{j} \in B_{j}, N_{t} \geq n\right] .
\end{aligned}
$$

There are two cases to consider in evaluating the inner summations of (5.19), namely, $i=j=n$ and $i<j=n$ (with $i=n>j$ being similar).

Consider $i=j=n$, for which the summands involve $A_{n} \cap B_{n}$. Because of the disjointness of $J_{1}$ and $J_{2}$, it follows from their definitions in (5.16) that

$$
\begin{aligned}
A_{n} & \cap B_{n} \\
& =\left\{\left(\frac{t}{M_{n-1}} J_{1}\right) \cap\left(1-\frac{t}{M_{n-1}} J_{2}\right)\right\} \cup\left\{\left(\frac{t}{M_{n-1}} J_{2}\right) \cap\left(1-\frac{t}{M_{n-1}} J_{1}\right)\right\} .
\end{aligned}
$$


Notice that the two sets in parentheses are disjoint and have the same Lebesgue measure. Thus

$$
\begin{aligned}
P\left[U_{n}\right. & \left.\in A_{n} \cap B_{n}, N_{t} \geq n\right] \\
& =2 P\left[U_{n} \in \frac{t}{M_{n-1}}\left\{J_{1} \cap\left(\frac{M_{n-1}}{t}-J_{2}\right)\right\}, M_{n-1}>t\right] \\
& =2 E\left(\frac{t}{M_{n-1}}\left|J_{1} \cap\left(\frac{M_{n-1}}{t}-J_{2}\right)\right| \mathbb{1}_{\left[M_{n-1}>t\right]}\right) \\
& \leq 2 E\left(\left|J_{1} \cap\left(\frac{M_{n-1}}{t}-J_{2}\right)\right| \mathbb{1}_{\left[M_{n-1}>t\right]}\right) .
\end{aligned}
$$

However, for any $v>1$,

$$
\left|J_{1} \cap\left(v-J_{2}\right)\right|=\int_{0}^{1} \mathbb{1}_{J_{1}}(x) \mathbb{1}_{J_{2}}(v-x) d x=\left(\mathbb{1}_{J_{1}} * \mathbb{1}_{J_{2}}\right)(v),
$$

the convolution of two indicator functions. By Fubini,

$$
\begin{aligned}
& P\left[U_{n}\right.\left.\in A_{n} \cap B_{n}, N_{t} \geq n\right] \\
& \quad \leq 2 \int_{0}^{1} \mathbb{1}_{J_{1}}(x) E\left\{\mathbb{1}_{J_{2}}\left(\frac{M_{n-1}}{t}-x\right) \mathbb{1}_{\left[M_{n-1}>t\right]}\right\} d x .
\end{aligned}
$$

Since $J_{2} \subset[0,1]$ and $0<x \leq 1$, the expectation in the integrand is equal to $P\left[M_{n-1} \in t L_{x}\right]$ where $L_{x}=\left(x+J_{2}\right) \cap(1,2]$. But

$$
P\left[M_{n-1} \in t L_{x}\right]=\int_{t L_{x}} d_{s} P\left[M_{n-1} \leq s\right]=\int_{t L_{x}} d_{s}\left(-P\left[N_{s} \geq n\right]\right) .
$$

Thus (5.21) yields

(5.22) $P\left[U_{n} \in A_{n} \cap B_{n}, N_{t} \geq n\right] \leq 2 \int_{0}^{1} \mathbb{1}_{J_{1}}(x) \int_{t L_{x}} d_{s}\left(-P\left[N_{s} \geq n\right]\right) d x$.

Substitution of this into (5.19) shows that the part of the summation for which $i=j=n$ satisfies

$$
\begin{aligned}
& 64 \sum_{n=1}^{\infty} n^{2} P\left[U_{n} \in A_{n} \cap B_{n}, N_{t} \geq n\right] \\
& \quad \leq 128 \int_{0}^{1} \mathbb{1}_{J_{1}}(x) \int_{t L_{x}} d_{s}\left(-\sum_{n=1}^{\infty} n^{2} P\left[N_{s} \geq n\right]\right) d x .
\end{aligned}
$$


However, the mass function

$$
\begin{aligned}
\Phi(s) & :=\sum_{n=1}^{\infty} n^{2} P\left[N_{s} \geq n\right] \\
& =\sum_{n=1}^{\infty} n^{2} \sum_{k=n}^{\infty} P\left[N_{s}=k\right]=\sum_{k=1}^{\infty} \frac{k(k+1)(2 k+1)}{6} P\left[N_{s}=k\right] \\
& =\frac{1}{3} E N_{s}^{3}+\frac{1}{2} E N_{s}^{2}+\frac{1}{6} E N_{s} .
\end{aligned}
$$

Since in the integration of (5.23), $s \in(t, 2 t]$, it follows that $s \leq 1 / 3$ whenever $t \leq 1 / 6$. In this case, then, the key Corollary 2.3 implies that

$$
\begin{aligned}
\Phi(s) & =\frac{1}{3}\left\{\kappa_{3}\left(N_{s}\right)+3 \kappa_{2}\left(N_{s}\right) E N_{s}+\left(E N_{s}\right)^{3}\right\}+\frac{1}{2}\left\{\kappa_{2}\left(N_{s}\right)+\left(E N_{s}\right)^{2}\right\}+\frac{1}{6} E N_{s} \\
& =\frac{c_{3}}{3 s}+\frac{c_{2}}{s}\left(\frac{2}{s}-1\right)+\frac{1}{3}\left(\frac{2}{s}-1\right)^{3}+\frac{c_{2}}{2 s}+\frac{1}{2}\left(\frac{2}{s}-1\right)^{2}+\frac{1}{6}\left(\frac{2}{s}-1\right) \\
& \equiv a_{0}+a_{1} s^{-1}+a_{2} s^{-2}+a_{3} s^{-3},
\end{aligned}
$$

say, and so $\Phi$ is differentiable with $-\Phi^{\prime}(s) \leq b s^{-4}$ for some constant $b$ for $s \in(t, 2 t]$. Substitution into (5.23) shows that the part of the summation in (5.19) with $i=j=n$ satisfies

$$
64 \sum_{n=1}^{\infty} n^{2} P\left[U_{n} \in A_{n} \cap B_{n}, N_{t} \geq n\right] \leq 128 b t^{-3}\left|J_{1}\right|\left|J_{2}\right| \leq C t^{-3}\left|J_{1}\right|\left|J_{2}\right|
$$

as desired whenever $0<t \leq 1 / 6$. Note that since $t\left(N_{t}+1\right) \geq 1$ always, $t^{-3} \leq$ $E\left(N_{t}+1\right)^{3} \leq 8 E N_{t}^{4}$.

Consider now $t>1 / 6$. To show that the measure determined by $\Phi$ remains dominated by Lebesgue measure over $(t, 1)$, it suffices to show that for any $k \geq 1$ and any $1 / 6<s<r<1, E\left(N_{s}^{k}-N_{r}^{k}\right) \leq b(r-s)$ for some constant $b$. To this end, for $1 / 6<s<r<1$ consider

$$
\begin{aligned}
N_{s}^{k}-N_{r}^{k} & =\left[N_{r}+\left(N_{s}-N_{r}\right)\right]^{k}-N_{r}^{k} \\
& =\sum_{l=0}^{k-1}\left(\begin{array}{l}
k \\
l
\end{array}\right) N_{r}^{l}\left(N_{s}-N_{r}\right)^{k-l} \leq C_{k} N_{r}^{k-1}\left(N_{s}-N_{r}\right)^{k}
\end{aligned}
$$

for some constant $C_{k}$. Moreover, by (5.1),

$$
\begin{aligned}
N_{s}-N_{r} & =\sum_{i: D_{r i}>s} N_{s / D_{r i}}^{(i)} \\
& =\sum_{i: D_{r i}>s}\left(N_{s / D_{r i}}^{(i)}-1\right)+K((s, r], r)
\end{aligned}
$$


in which the superscript $i$ indexes independent processes as in (5.1); see also the proof of Lemma 4.3. Thus, by Minkowski's inequality,

$$
\begin{aligned}
E\left(\left(N_{s}-N_{r}\right)^{k} \mid \mathcal{F}_{r}\right) & \leq\left\{\sum_{i: D_{r i}>s}\left\{E\left(N_{s / D_{r i}}^{(i)}-1\right)^{k}\right\}^{1 / k}+K((s, r], r)\right\}^{k} \\
& \leq\left\{K((s, r], r)\left\{E\left(N_{s / r}-1\right)^{k}\right\}^{1 / k}+K((s, r], r)\right\}^{k} \\
& \leq\{K((s, r], r)\}^{k}\left\{\left(E\left(N_{s / r}-1\right)^{k}\right)^{1 / k}+1\right\}^{k},
\end{aligned}
$$

in which $\mathcal{F}_{r}=\sigma\left(D_{r i}: 1 \leq i \leq N_{r}+1\right)$ as introduced in Section 3. Since to show the a.e. differentiability of $\Phi$ it suffices to consider $r-s$ small, assume without loss of generality that $r-s<1 / 6$, implying that $s / r>1 / 2$; recall that $1 / 6<s<r<1$. Hence, the second factor in the last expression is (by Lemma 2.1) bounded, and so

$$
\begin{aligned}
E\left(\left(N_{s}-N_{r}\right)^{k} \mid \mathcal{F}_{r}\right) & \leq C_{k}\{K((s, r], r)\}^{k} \\
& \leq C_{k} N_{r}^{k} \mathbb{1}_{\left[N_{s}>N_{r}\right]},
\end{aligned}
$$

where, here and in the following, $C_{k}$ is used generically to denote constants depending only upon $k$. Therefore, (5.26) yields

$$
E N_{s}^{k}-E N_{r}^{k} \leq C_{k} E\left(N_{r}^{2 k-1} \mathbb{1}_{\left[N_{s}>N_{r}\right]}\right) .
$$

The event $\left[N_{s}>N_{r}\right]=[K((s, r], r)>0]$ is the event that at least one of the first $N_{r}$ splits resulted in a spacing in $(s, r]$. As for the proof of (5.12) above but with $r$ in place of $t$, let $\eta_{j}$ be the number of spacings formed by the $j$ th splitting that have lengths in $r J \equiv(s, r]$ with $J=(s / r, 1]$. Then

$$
\left[N_{s}>N_{r}\right] \subset \bigcup_{j=1}^{\infty}\left[\eta_{j}>0, N_{r} \geq j\right]
$$

so that

$$
E\left(N_{r}^{2 k-1} \mathbb{1}_{\left[N_{s}>N_{r}\right]}\right) \leq \sum_{j=1}^{\infty} E\left(N_{r}^{2 k-1} \mathbb{1}_{\left[\eta_{j}>0, N_{r} \geq j\right]}\right) .
$$

However, conditionally given $\left\{D_{j-1, i}: 1 \leq i \leq j\right\}$ and $\mathbb{1}_{\left[N_{r} \geq j\right]}, N_{r}$ is stochastically dominated as follows:

$$
N_{r} \stackrel{L}{=} j+\sum_{i: D_{j, i}>r} N_{r / D_{j, i}}^{(i)} \stackrel{\text { stoch }}{\preceq} j+\sum_{i=1}^{[1 / r]} N_{r}^{(i)},
$$

since after $j$ splittings, the splitting process continues independently in the intervals whose lengths, $D_{j, i}$, still exceed $r$, and since the number of such intervals 
does not exceed $1 / r$. Note in particular that this stochastic bound does not depend upon $U_{j}$, the $j$ th splitting uniform r.v., nor, therefore, upon $\eta_{j}$. Thus

$$
\begin{aligned}
& E\left(N_{r}^{2 k-1} \mathbb{1}_{\left[\eta_{j}>0, N_{r} \geq j\right]}\right) \\
& \quad \leq E\left(j+\sum_{i=1}^{[1 / r]} N_{r}^{(i)}\right)^{2 k-1} P\left[\eta_{j}>0, N_{r} \geq j\right] \\
& \quad \leq\left(j+\frac{1}{r}\left(E N_{r}^{2 k-1}\right)^{1 /(2 k-1)}\right)^{2 k-1} P\left[\eta_{j}>0, N_{r} \geq j\right] \\
& \quad \leq C_{k} j^{2 k-1} P\left[\eta_{j}>0, N_{r} \geq j\right],
\end{aligned}
$$

in which the second inequality utilizes Minkowski's inequality and Lemma 2.1; recall $r>1 / 6$. Moreover, as in the proof of (5.12),

$$
\begin{aligned}
P\left[\eta_{j}>0, N_{r} \geq j\right] & \leq 2 P\left[U_{j} M_{j-1} \in(s, r], N_{r} \geq j\right] \\
& =2 E\left(\left(r / M_{j-1}\right)(1-s / r) \mathbb{1}_{\left[N_{r} \geq j\right]}\right) \\
& \leq \frac{2}{r}(r-s) P\left[N_{r} \geq j\right] .
\end{aligned}
$$

Thus (5.29), (5.31) and (5.32) applied to (5.28) yields

$$
\begin{aligned}
E N_{s}^{k}-E N_{r}^{k} & \leq C_{k}(r-s) \sum_{j=1}^{\infty} j^{2 k-1} P\left[N_{r} \geq j\right] \\
& \leq C_{k}(r-s) E N_{r}^{2 k} \leq C_{k}(r-s)
\end{aligned}
$$

by Lemma 2.1 for all $k \geq 1$ and $1 / 6<s<r<1$ with $r-s<1 / 6$. By (5.24) this shows that a bounded $\Phi^{\prime}$ exists a.e. over $(1 / 6,1)$. Hence (5.23) yields

$$
\begin{aligned}
64 \sum_{n=1}^{\infty} n^{2} P\left[U_{n} \in A_{n} \cap B_{n}, N_{t} \geq n\right] & \leq C \int_{0}^{1} \mathbb{1}_{J_{1}}(x)\left|t L_{x}\right| d x \\
& \leq C\left|J_{1}\right|\left|J_{2}\right|
\end{aligned}
$$

as desired when $t>1 / 6$. This and (5.25) complete the bounding of the terms in (5.19) with $i=j=n$.

To compute a bound for the other terms of (5.19) in which $i<j=n$, observe that

$$
\begin{aligned}
P\left[U_{i}\right. & \left.\in A_{i}, U_{n} \in B_{n}, N_{t} \geq n\right] \\
& =E\left\{E\left(\mathbb{1}_{\left[U_{n} \in B_{n}\right]} \mid M_{n-1}, U_{i} \in A_{i}, N_{t} \geq n\right) \mathbb{1}_{\left[U_{i} \in A_{i}\right]} \mathbb{1}_{\left[N_{t} \geq n\right]}\right\} \\
& \leq E\left\{\left(2 t / M_{n-1}\right)\left|J_{2}\right| \mathbb{1}_{\left[U_{i} \in A_{i}, N_{t} \geq n\right]}\right\} \\
& \leq 2\left|J_{2}\right| P\left[U_{i} \in A_{i}, N_{t} \geq i, N_{t} \geq n\right]
\end{aligned}
$$


where the insertion of $\left[N_{t} \geq i\right]$ changes nothing since $n>i$. To compute the remaining probability, notice that when $U_{i} \in A_{i}$, (5.16) implies that at least one of $U_{i} M_{i-1}$ or $\left(1-U_{i}\right) M_{i-1}$ is in $t J_{1}$, and thus is less than or equal to $t$. Hence, at least one of the two spacings formed by the $i$ th splitting is never split again during the next $N_{t}-i$ splittings.

Given $\left\{D_{i-1, k}: 1 \leq k \leq i\right\},\left[N_{t} \geq i\right]$ and $\left[U_{i} \in t J_{1} / M_{i-1}\right]$, the conditional distribution of $N_{t}$ is, as in (5.30), that of

$$
i+\sum_{k: D_{i-1, k}<M_{i-1}} N_{t / D_{i-1, k}}^{(k)}+N_{t /\left(1-U_{i}\right) M_{i-1}}^{(0)}
$$

where the $N^{(k)}$ are independent copies of $N$. Clearly the last term of (5.36) does not exceed $N_{t / M_{i-1}}^{(0)}$ so that (5.36) is stochastically smaller than

$$
i+\sum_{k=1}^{i} N_{t / D_{i-1, k}}^{(k)} .
$$

This in turn has the same distribution as the conditional distribution of $N_{t}+1$ given $\left\{D_{i-1, k}: 1 \leq k \leq i\right\}$ and $\left[N_{t} \geq i\right]$. The same result holds if we had assumed that $1-U_{i} \in t J_{1} / M_{i-1}$, and hence if $U_{i} \in A_{i}$. It follows that

$$
\begin{aligned}
P\left[U_{i}\right. & \left.\in A_{i}, N_{t} \geq i, N_{t} \geq n\right] \\
& =E\left\{P\left[N_{t} \geq n \mid\left\{D_{i-1, k}: 1 \leq k \leq i\right\}, U_{i} \in A_{i}, N_{t} \geq i\right] \mathbb{1}_{\left[U_{i} \in A_{i}, N_{t} \geq i\right]}\right\} \\
& \leq E\left\{P\left[N_{t} \geq n-1 \mid\left\{D_{i-1, k}: 1 \leq k \leq i\right\}, N_{t} \geq i\right] \mathbb{1}_{\left[U_{i} \in A_{i}, N_{t} \geq i\right]}\right\} \\
& =E P\left[N_{t} \geq n-1 \mid N_{t} \geq i, M_{i-1}\right] \mathbb{1}_{\left[N_{t} \geq i\right]} P\left[U_{i} \in A_{i} \mid N_{t} \geq i, M_{i-1}\right] .
\end{aligned}
$$

However,

$$
P\left[U_{i} \in A_{i} \mid N_{t} \geq i, M_{i-1}\right] \leq 2\left(t / M_{i-1}\right)\left|J_{1}\right| \leq 2\left|J_{1}\right| .
$$

Thus, for $1 \leq i \leq n-1$,

$$
\begin{aligned}
P\left[U_{i} \in A_{i}, N_{t} \geq i, N_{t} \geq n\right] & \leq 2\left|J_{1}\right| P\left[N_{t} \geq n-1 \mid N_{t} \geq i\right] P\left[N_{t} \geq i\right] \\
& \leq 2\left|J_{1}\right| P\left[N_{t} \geq n-1\right] .
\end{aligned}
$$

In view of (5.35) this implies that the sum of the terms of (5.19) with $i<j=n$ is bounded by

$$
\begin{aligned}
& 256\left|J_{1}\right|\left|J_{2}\right| \sum_{n=1}^{\infty} \sum_{i=1}^{n-1} n i P\left[N_{t} \geq n-1\right] \\
& =128\left|J_{1}\right|\left|J_{2}\right| \sum_{n=1}^{\infty} n^{2}(n-1) P\left[N_{t} \geq n-1\right] \\
& =128\left|J_{1}\right|\left|J_{2}\right| E\left\{\sum_{n=1}^{N_{t}+1} n^{2}(n-1)\right\} \leq 64\left|J_{1}\right|\left|J_{2}\right| E\left(N_{t}+1\right)^{4} .
\end{aligned}
$$


Together with (5.25) and (5.34) this completes the proof of (5.13) and hence of Lemma 5.3.

Return now to the proof of Lemma 5.2 in the remaining case of $t>1 / 4$, or more generally, when $t$ is bounded away from 0 . Observe that since for any $t$, $W\left(J_{i}, t\right)=(t / 2)^{1 / 2}\left[K\left(t J_{i}, t\right)-2\left|J_{i}\right| / t\right]$, direct expansion in (5.7) yields

$$
\begin{aligned}
& E\left[W\left(J_{1}, t\right) W\left(J_{2}, t\right)\right]^{2} \\
& =\frac{t^{2}}{4} E\left\{K^{2}\left(t J_{1}, t\right)-\frac{4}{t}\left|J_{1}\right| K\left(t J_{1}, t\right)+\frac{4}{t^{2}}\left|J_{1}\right|^{2}\right\} \\
& \quad \times\left\{K^{2}\left(t J_{2}, t\right)-\frac{4}{t}\left|J_{2}\right| K\left(t J_{2}, t\right)+\frac{4}{t^{2}}\left|J_{2}\right|^{2}\right\} \\
& \leq \frac{t^{2}}{4} E\left\{\left[K\left(t J_{1}, t\right) K\left(t J_{2}, t\right)\right]^{2}+\frac{16}{t^{2}}\left|J_{1}\right|\left|J_{2}\right|\left[K\left(t J_{1}, t\right) K\left(t J_{2}, t\right)\right]\right. \\
& \left.\quad+\frac{16}{t^{4}}\left|J_{1}\right|^{2}\left|J_{2}\right|^{2}+\frac{4}{t^{2}}\left|J_{1}\right|^{2} K^{2}\left(t J_{2}, t\right)+\frac{4}{t^{2}}\left|J_{2}\right|^{2} K^{2}\left(t J_{1}, t\right)\right\} .
\end{aligned}
$$

Thus (5.7) follows directly from Lemma 5.3 whenever $t$ is bounded away from 0 . This completes the proof of Lemma 5.2.

Tightness clearly follows from Lemma 5.2; Chebyshev's inequality implies that for any $\lambda>0$ and disjoint adjacent intervals $J_{1}$ and $J_{2}$ in $[0,1]$,

$$
\begin{aligned}
& P\left[\left|W\left(J_{1}, t\right)\right|>\lambda,\left|W\left(J_{2}, t\right)\right|>\lambda\right] \\
& \quad \leq P\left[\left|W\left(J_{1}, t\right) W\left(J_{2}, t\right)\right|>\lambda^{2}\right] \leq \lambda^{-4} C\left|J_{1}\right|\left|J_{2}\right| .
\end{aligned}
$$

Theorem 15.6 of Billingsley (1968), then suffices, together with the finitedimensional limits established earlier in Lemma 5.1, to prove the following main result.

THEOREM 5.4. The stopped processes $W(\cdot, t)$ converge weakly in $D[0,1]$ as $t \rightarrow 0$ to a mean zero Gaussian process $W(\cdot)$ with covariance given by (7.10). Moreover, by (1.20), the stopped empirical processes of the relative spacings satisfy $V(\cdot, t) \rightarrow_{L} V$ where $V(y)=W(y)-y W(1)$, a mean zero Gaussian process with covariance given by (7.12).

6. Weak convergence of the $\boldsymbol{V}_{\boldsymbol{n}}$-processes. Consider the empirical process of the relative spacings, $\left\{D_{n i} / M_{n} ; 1 \leq i \leq n+1\right\}$, defined in (1.17) by

$$
V_{n}(y)=\sqrt{n+1}\left\{G_{n}^{*}\left((n+1) M_{n} y\right)-y\right\}, \quad 0 \leq y \leq 1 .
$$


The weak convergence of the related stopped process

$$
V(y, s)=\frac{2}{s\left(N_{s}+1\right)}\{W(y, s)-y W(1, s)\}
$$

for $0 \leq y \leq 1$ and $0<s \leq 1$ is given in Theorem 5.4, namely,

$$
V(\cdot, s) \stackrel{L}{\rightarrow} W(\cdot)-(\cdot) W(1)=V(\cdot)
$$

in $D[0,1]$ as $s \rightarrow 0$, with the limit process $V$ being a mean zero Gaussian process with covariance given in (7.12).

By the above definitions, one may write

$$
V_{n}(y)=\left(M_{n}(n+1) / 2\right)^{1 / 2} V\left(y, M_{n}\right),
$$

which means that the desired weak convergence of $V_{n}$ will be established once it is established for $W\left(\cdot, M_{n}\right)$.

To show the latter, we will establish that as $n \rightarrow \infty$,

$$
W(\cdot, 2 / n)-W\left(\cdot, M_{n}\right) \stackrel{L}{\rightarrow} 0
$$

in $D[0,1]$. From this, the limit process for $W\left(\cdot, M_{n}\right)$ is seen to be that of $W(\cdot, 2 / n)$, namely, $W$.

The first step is to show that for any $\varepsilon>0$, there exists $n_{\varepsilon}$ and $L=L_{\varepsilon}>0$ such that

$$
P\left(\left|M_{n}-\frac{2}{n}\right| \geq L n^{-3 / 2}\right) \leq \varepsilon
$$

for all $n \geq n_{\varepsilon}$. To see this, observe that the expression following (4.14) states that for any $A>0$,

$$
P\left(M_{n}-\frac{2}{n}>\left(\frac{n}{2}-A n^{1 / 2}\right)^{-1}-\frac{2}{n}\right)=P\left(M_{n}>\left(\frac{n}{2}-A n^{1 / 2}\right)^{-1}\right) \leq \frac{c}{8 A^{2}}
$$

for all $n$ sufficiently large. But, for $A=L / 8$,

$$
\left(\frac{n}{2}-A n^{1 / 2}\right)^{-1}-\frac{2}{n}=\frac{2}{n}\left[\left(1-2 A n^{-1 / 2}\right)^{-1}-1\right]<8 A n^{-3 / 2}=L n^{-3 / 2}
$$

for all $n$ sufficiently large, implying that

$$
P\left(M_{n}-\frac{2}{n} \geq L n^{-3 / 2}\right) \leq \frac{8 c}{L^{2}}
$$

for all $n$ sufficiently large. Together with (4.14)'s analogous bound for small values of $M_{n}-2 / n$, this proves (6.6). Alternatively, one may use a standard renewal theory argument to obtain the limit law for $n^{3 / 2}\left(M_{n}-2 / n\right)$ from that of $N_{s}$ since $\left(M_{n}>s\right)=\left(N_{s}>n\right)$; specifically, one obtains from Corollary 3.3 that $n^{1 / 2}\left\{n M_{n} / 2-1\right\}$ has the same asymptotic normal distribution $N\left(0, \sigma^{2}\right)$ as does $(2 / t)^{1 / 2}\left(t N_{t} / 2-1\right)$. 
In view of (6.6), the proof of (6.5) will be complete if we can show that for every integer $L>0$

$$
\sup _{s:|t-s| \leq L t^{3 / 2}} \sup _{0 \leq y \leq 1}|W(y, s)-W(y, t)| \stackrel{P}{\rightarrow} 0
$$

as $t \rightarrow 0$. To handle the supremum over $s$ in the above, equip the interval $I_{t}=$ $\left[t-L t^{3 / 2}, t+L t^{3 / 2}\right]$ with the grid of $2 L^{2}+1$ equally spaced points $s_{i} \equiv s_{i}(t)=$ $t+(-L+i / L) t^{3 / 2}, i=0,1, \ldots, 2 L^{2}$. Note that $s_{i+1}-s_{i}=t^{3 / 2} / L$ for each $i$. Assume without loss of generality that $t<(2 L)^{-2}$ to insure that each $s_{i} \in(0,1)$ and that $t / s_{i}<2$ for each $i$. To prove (6.7) it obviously suffices to show that for every positive integer $L \geq 2$,

$$
\sup _{0 \leq y \leq 1}\left|W(y, t)-W\left(y, s_{i}\right)\right| \stackrel{P}{\rightarrow} 0 \quad \text { for } i=0,1, \ldots, 2 L^{2}
$$

as $t \rightarrow 0$, and that for every $\varepsilon>0, \delta>0$, there exist $L>0$ and $t^{*}>0$ such that for $t<t^{*}$,

$$
P\left(\max _{0 \leq i<2 L^{2}} \sup _{s_{i} \leq s \leq s_{i+1}} \sup _{0 \leq y \leq 1}\left|W(y, s)-W\left(y, s_{i}\right)\right|>\delta\right)<\varepsilon .
$$

To prove (6.8), observe first that known characterizations of tightness for $D[0,1]$-valued processes imply that the processes formed by summing two tight families of processes are also tight; compare Theorem 15.2 of Billingsley (1968) and check that the $D[0,1]$ modulus $w_{x}^{\prime}(\delta)$ satisfies the following: For any $\varepsilon>0$ and $\delta>0$, there exists $\delta^{*} \equiv \delta^{*}(\varepsilon, \delta)$ for which

$$
w_{f+g}^{\prime}\left(\delta^{*}\right) \leq w_{f}^{\prime}(\delta)+w_{g}^{\prime}(\delta)+2 \varepsilon .
$$

This follows by first choosing $\delta$-partitions that approximate the moduli $w_{f}^{\prime}(\delta)$ and $w_{g}^{\prime}(\delta)$ to within $\varepsilon$, and then use the refinement of these two partitions to obtain an upper bound for $w_{f+g}^{\prime}\left(\delta^{*}\right)$ in which $\delta^{*}$ is the span of this refinement. In view of Theorem 5.4 this means that the family of processes $\left\{W(\cdot, t)-W\left(\cdot, s_{i}\right): t \in(0,1]\right\}$ is tight. Thus, to show $W(\cdot, t)-W\left(\cdot, s_{i}\right) \rightarrow_{L} 0$ as $t \rightarrow 0$, and hence the uniform convergence in probability to zero that is expressed in (6.8), it suffices to show $W(y, t)-W\left(y, s_{i}\right) \rightarrow_{P} 0$ for each fixed $y \in[0,1]$. This we do by establishing the following lemma.

LEMMA 6.1. For $0<s \leq 1 / 2$ and $0<\lambda<1$,

$$
\sup _{0 \leq y \leq 1} E[W(y, s)-W(y, \lambda s)]^{2}=O(1-\lambda) \text {. }
$$

PROOF. From (7.9) and (7.10), we have, for any $0 \leq y, z \leq 1$ and $0<s \leq 1 / 2$,

$$
\operatorname{Cov}(W(y, s), W(z, s))=\frac{1}{2} \alpha(y \wedge z, y \vee z) \equiv A(y, z)+y \wedge z
$$


in which the non-Brownian portion of the covariance,

$$
\begin{aligned}
A(y, z)= & -6 y z+2 y z\left\{(1+y)^{-1}+(1+z)^{-1}\right\}+2 y \ln (1+z) \\
& +2 z \ln (1+y)+(y+z-1)^{+}\left(1-(y+z)^{-1}\right),
\end{aligned}
$$

is a symmetric function with uniformly bounded partial derivatives over $[0,1]^{2}$. By (5.3),

$$
\begin{aligned}
& E\{W(y, \lambda s) W(y, s)\} \\
& =\lambda^{1 / 2} E\{W(\lambda y, s) W(y, s)\} \\
& \quad+2 y \lambda^{-1 / 2} E\left\{\left[W(1, s)-\lambda W(\lambda, s)-\int_{\lambda}^{1} W(x, s) d x\right] W(y, s)\right\}
\end{aligned}
$$

where integration by parts has been applied to the last term of (5.3). Consequently, (6.10) implies that

$$
\begin{aligned}
E\{W(y, \lambda s) W(y, s)\} & \\
= & \sqrt{\lambda}\{A(\lambda y, y)+\lambda y\}+\frac{2 y}{\sqrt{\lambda}} A(y, 1) \\
& -2 y \sqrt{\lambda} A(y, \lambda)-\frac{2 y}{\sqrt{\lambda}} \int_{\lambda}^{1} A(x, y) d x \\
& +\frac{2 y^{2}}{\sqrt{\lambda}}-2 y \sqrt{\lambda}(y \wedge \lambda)-\frac{2 y}{\sqrt{\lambda}} \int_{\lambda}^{1}(x \wedge y) d x .
\end{aligned}
$$

Hence,

$$
\begin{aligned}
& E[W(y, s)-W(y, \lambda s)]^{2} \\
& =2 A(y, y)+2 y-2 E\{W(y, \lambda s) W(y, s)\} \\
& =2\{A(y, y)-\sqrt{\lambda} A(\lambda y, y)\}+\frac{4 y}{\sqrt{\lambda}}\{\lambda A(y, \lambda)-A(y, 1)\} \\
& \quad+\frac{4 y}{\sqrt{\lambda}} \int_{\lambda}^{1} A(x, y) d x+2 y\left(1-\lambda^{3 / 2}\right) \\
& \quad+\frac{4 y}{\sqrt{\lambda}}\{\lambda(y \wedge \lambda)-y\}+\frac{4 y}{\sqrt{\lambda}} \int_{\lambda}^{1}(x \wedge y) d x .
\end{aligned}
$$

It follows from $(6.11)$ that $A(\cdot, \cdot)$ and its first-order partials are bounded, thereby insuring that the first three terms above are of order $O(1-\lambda)$. The last three terms, not involving $A(\cdot, \cdot)$, are easily checked to be of the desired order as well, thereby completing the proof. 
By taking $s=s_{i}$ and $\lambda=t / s_{i}$ for $i=0,1, \ldots, 2 L^{2}$, it follows from Lemma 6.1 that $W(y, t)-W\left(y, s_{i}\right) \rightarrow{ }_{P} 0$ for each $y$ as $t \rightarrow 0$; note that for each fixed $L>0$,

$$
1-\lambda \equiv \frac{s_{i}-t}{s_{i}} \leq \frac{L \sqrt{t}}{1-L \sqrt{t}}=O(\sqrt{t}) \rightarrow 0
$$

as $t \rightarrow 0$. This completes the proof of (6.8).

The proof of (6.9) uses the following inequalities. For $0<u \leq s \leq v \leq 1$, we have [cf. (1.5)]

$$
K(y u, v) \leq K(y s, v) \leq K(y s, s) \leq K(y s, u) \leq K((y v) \wedge u, u)
$$

and so by (1.18), with $\rho \equiv u / v$ and $0 \leq y \leq 1$,

$$
\begin{aligned}
\sqrt{\rho} W & (\rho y, v)-\sqrt{\frac{2}{v}} \frac{1}{\sqrt{\rho}}\left(1-\rho^{2}\right) \\
& \leq W(y, s) \\
& \leq \frac{1}{\sqrt{\rho}} W\left(\frac{y}{\rho} \wedge 1, \rho v\right)+\sqrt{\frac{2}{v}}\left\{\frac{1}{\rho}\left(\frac{y}{\rho} \wedge 1\right)-y\right\} \\
& \leq \frac{1}{\sqrt{\rho}} W\left(\frac{y}{\rho} \wedge 1, \rho v\right)+\sqrt{\frac{2}{v}}\left(\rho^{-2}-1\right) .
\end{aligned}
$$

For application of these bounds to (6.9), take $v=s_{i+1}$ and $u=s_{i}$ so that $\rho=$ $s_{i} / s_{i+1}$. Observe that by definition,

$$
0 \leq 1-\rho=\frac{s_{i+1}-s_{i}}{s_{i+1}}=\frac{\sqrt{t} / L}{1+(-L+(i+1) / L) \sqrt{t}} \leq \frac{\sqrt{t} / L}{1-L \sqrt{t}} .
$$

Hence, since we have assumed $t<(2 L)^{-2}$ and $L \geq 2$, then $1-\rho<2 \sqrt{t} / L$ and $\rho>3 / 4$. It follows that the nonrandom terms in the bounds of (6.15) are bounded in absolute value by $16 / L$. Consequently, for $s_{i} \leq s \leq s_{i+1}$ and $0 \leq y \leq 1$, we obtain the following uniform bounds for (6.9), in which we write $\|\cdot\|$ for the supremum over $[0,1]$ and $w_{f}(\delta)=\sup \{|f(u)-f(v)|: 0 \leq u \leq v \leq u+\delta \leq 1\}$ for the usual modulus of continuity:

$$
\begin{aligned}
W(y, s)-W\left(y, s_{i}\right) & \leq \frac{1}{\sqrt{\rho}} W\left(\frac{y}{\rho} \wedge 1, s_{i}\right)-W\left(y, s_{i}\right)+\frac{16}{L} \\
& =\left(\rho^{-1 / 2}-1\right) W\left(\frac{y}{\rho} \wedge 1, s_{i}\right)+W\left(\left(y, \frac{y}{\rho} \wedge 1\right], s_{i}\right)+\frac{16}{L} \\
& \leq(1-\rho)\left\|W\left(\cdot, s_{i}\right)\right\|+w_{W\left(\cdot, s_{i}\right)}\left(\rho^{-1}-1\right)+\frac{16}{L} \\
& \leq \frac{2 \sqrt{t}}{L}\left\|W\left(\cdot, s_{i}\right)\right\|+w_{W\left(\cdot, s_{i}\right)}\left(\frac{2 \sqrt{t}}{L}\right)+\frac{16}{L}
\end{aligned}
$$


and

$$
\begin{aligned}
W(y, s)-W\left(y, s_{i}\right) \geq & \sqrt{\rho} W\left(\rho y, s_{i+1}\right)-W\left(y, s_{i}\right)-\frac{16}{L} \\
= & \left(\rho^{1 / 2}-1\right) W\left(\rho y, s_{i+1}\right)-W\left(y(\rho, 1], s_{i+1}\right) \\
& +W\left(y, s_{i+1}\right)-W\left(y, s_{i}\right)-\frac{16}{L} \\
\geq & -(1-\rho)\left\|W\left(\cdot, s_{i+1}\right)\right\|-w_{W\left(\cdot, s_{i+1}\right)}(1-\rho) \\
& -\left\|W\left(\cdot, s_{i+1}\right)-W\left(\cdot, s_{i}\right)\right\|-\frac{16}{L} \\
\geq & -\frac{2 \sqrt{t}}{L}\left\|W\left(\cdot, s_{i+1}\right)\right\|-w_{W\left(\cdot, s_{i+1}\right)}\left(\frac{2 \sqrt{t}}{L}\right) \\
& -\left\|W\left(\cdot, s_{i+1}\right)-W\left(\cdot, s_{i}\right)\right\|-\frac{16}{L} .
\end{aligned}
$$

Since the maximum discontinuity of $W(\cdot, s)$ is $(s / 2)^{1 / 2}$, the limiting process, $W$, in Theorem 5.4 is continuous so that for every $i$ and $L, w_{W(\cdot, s)}(2 \sqrt{t} /$ $L) \rightarrow{ }_{P} 0$ and $\left\|W\left(\cdot, s_{i}\right)\right\|=O_{p}(1)$. Moreover, a similar argument to that used earlier to prove (6.9) suffices to show that $\left\|W\left(\cdot, s_{i+1}\right)-W\left(\cdot, s_{i}\right)\right\| \rightarrow_{P} 0$ for each $i$. Therefore, for each fixed $L$,

$$
\max _{0 \leq i<2 L^{2}} \sup _{s_{i} \leq s \leq s_{i+1}}\left\|W(\cdot, s)-W\left(\cdot, s_{i}\right)\right\|=o_{p}(1)+\frac{16}{L},
$$

which establishes (6.9). This completes the proof of (6.8) and (6.9), and hence of (6.7) and (6.5), thereby proving:

THEOREM 6.2. The empirical processes of the relative spacings, $V_{n}: n \geq 1$, converge weakly in $D(0,1)$ to the mean zero Gaussian process $V$ with covariance function given in (7.12).

7. The covariance of the spacings processes. The covariance functions of the limiting empirical processes for the normalized spacings are only given implicitly in the above in the sense that they are expressible in terms of constants from Theorem 2.2 for $D(t)=K(t J, t)$. An explicit expression for these covariances is now derived, thereby completing the characterization of the limiting process.

The basic function is the covariance of $K(\cdot, s)$. For $0 \leq x \leq y \leq s$ and $0 \leq s \leq 1$, set

$$
\begin{aligned}
& c(x, y, s)=\operatorname{Cov}(K(x, s), K(y, s)), \\
& C(u, v, s)=c(u s, v s, s) \quad \text { for } 0 \leq u \leq v
\end{aligned}
$$


Since $K$ satisfies the representation (1.10), we already know much of the structure of the covariance because of Theorem 2.4; note that $\mathbf{D}(t)=(K(u t, t), K(v t, t))$ satisfies the hypotheses of Theorem 2.4. Thus for $0 \leq t \leq 1 / 2$ the second mixed cumulant, $\kappa_{1,1}(t) \equiv \kappa_{1,1}(K(u t, t), K(v t, t))=C(u, v, t)$, is proportional to $t^{-1}$ for each $u$ and $v$. Specifically,

$$
C(u, v, t)=(2 t)^{-1} C\left(u, v, \frac{1}{2}\right) \quad \text { for } 0<t \leq \frac{1}{2} .
$$

What remains to be done is to compute the actual proportionality constants and this is what is done below.

In view of (1.10), with $K^{*}$ denoting an independent copy of $K$,

$$
\begin{array}{r}
c(x, y, s)=\operatorname{Cov}\left(K\left(\frac{x}{U}, \frac{s}{U}\right)+K^{*}\left(\frac{x}{1-U}, \frac{s}{1-U}\right),\right. \\
\left.K\left(\frac{y}{U}, \frac{s}{U}\right)+K^{*}\left(\frac{y}{1-U}, \frac{s}{1-U}\right)\right)
\end{array}
$$

$$
\begin{aligned}
= & 2 \int_{0}^{1} c(x / u, y / u, s / u) d u+2 \int_{0}^{1} \mu(x / u, s / u) \mu(y / u, s / u) d u \\
& +2 \int_{0}^{1} \mu(x / u, s / u) \mu(y /(1-u), s /(1-u)) d u-\mu(x, s) \mu(y, s)
\end{aligned}
$$

where $\mu(x, s)=E K(x, s)$. This uses the following easy computation: if $U$ is a Unif $(0,1)$ r.v. independent of processes $X(\cdot)$ and $Y(\cdot)$, then whenever the integrals make sense,

$$
\begin{aligned}
\operatorname{Cov} & X(U), Y(U)) \\
& =\int_{0}^{1} E X(u) Y(u) d u-E X(U) E Y(U) \\
& =\int_{0}^{1}\{\operatorname{Cov}(X(u), Y(u))+E X(u) E Y(u)\} d u-E X(U) E Y(U) .
\end{aligned}
$$

[In applying this to (7.3) we use the conditional independence of the summands making up $X(u)$ and $Y(u)$.]

In view of the evaluation of $\mu$ in (1.8), the second integral in (7.3) is

$$
\begin{aligned}
\int_{0}^{1} \mu(x / u, s / u) \mu(y / u, s / u) d u & =\int_{0}^{s} \varepsilon(x-u) \varepsilon(y-u) d u+\int_{s}^{1} \frac{2 x u}{s^{2}} \frac{2 y u}{s^{2}} d u \\
& =x+4 x y / 3 s^{4}-4 x y / 3 s .
\end{aligned}
$$

To evaluate the third integral of (7.3), note first that by (1.8), the integrand is zero when either $x<u \leq s$ or $1-s \leq u<1-y$. Upon applying (1.8) appropriately to the integrand over the remaining intervals of integration $0<u \leq x \wedge(1-s)$, $s \vee(1-y)<u \leq 1,1-y<u \leq x$ (when $x+y>1$ ) and $s<u \leq(1-s)$ (when 
$s<1 / 2$ ), one obtains

$$
\begin{aligned}
\int_{0}^{1} \mu(x / u, s / u) \mu\left(\frac{y}{1-u}, \frac{s}{1-u}\right) d u \\
=\left(x / s^{2}\right)\left(1-\{s \vee(1-y)\}^{2}\right) \\
\quad+\left(y / s^{2}\right)\left(1-\{s \vee(1-x)\}^{2}\right)+(x+y-1)^{+} \\
\quad+\mathbb{1}_{[s<1 / 2]} \frac{2 x y}{3 s^{4}}\left(4 s^{3}-6 s^{2}+1\right) .
\end{aligned}
$$

Together with (1.8) this allows us to evaluate $c(x, y, s)$ for $0 \leq x \leq y \leq s$ and $0<s \leq 1$ by means of (7.3). Substituting $x=u s$ and $y=v s$ we find that, for $0 \leq u \leq v \leq 1$ and $0<s \leq 1$,

$$
\begin{aligned}
C(u, v, s)= & 2 s \int_{s}^{1} C(u, v, w) w^{-2} d w+2 u s-4 u v / 3 s^{2}-8 u v s / 3 \\
& +(2 u / s)\left[1-\{s \vee(1-v s)\}^{2}\right]+(2 v / s)\left[1-\{s \vee(1-u s)\}^{2}\right] \\
& +2[(u+v) s-1]^{+}+\mathbb{1}_{[s<1 / 2]} \frac{4 u v}{3 s^{2}}\left(4 s^{3}-6 s^{2}+1\right) .
\end{aligned}
$$

This expression shows that $C(u, v, \cdot)$ is continuous on $(0,1)$ (recall that there is a discontinuity at $s=1)$ and is differentiable for all $s$ except possibly at the five values: $1 / 2 \leq(1+v)^{-1} \leq(1+u)^{-1} \leq[(u+v) \wedge 1]^{-1} \leq 1$. Dividing by $\mathrm{s}$, then differentiating with respect to $s$ and finally multiplying by $s^{2}$ shows that

$$
\begin{aligned}
\frac{d}{d s}\{s & C(u, v, s)\} \\
= & \frac{4 u v}{s^{2}}-\frac{4 u}{s} \mathbb{1}_{[s(1+v) \geq 1]}-\frac{4 v}{s} \mathbb{1}_{[s(1+u) \geq 1]}-4 u v \mathbb{1}_{[s(1+v)<1]} \\
& \quad-4 u v \mathbb{1}_{[s(1+u)<1]}+2 \mathbb{1}_{[s(u+v) \geq 1]}+4 u v\left(2-s^{-2}\right) \mathbb{1}_{[s<1 / 2]}
\end{aligned}
$$

for all but those five exceptional points. [The reader may note that the right-hand side of this expression is zero for $0<s<1 / 2$, thereby leading to an alternate proof that $s C(u, v, s)$ is constant over that range as stated in (7.2).] To obtain the proportionality constant of (7.2), we integrate the above from $s=1 / 2$ to $s=1-$ to obtain

$$
\begin{aligned}
& C(u, v, 1-)-\frac{1}{2} C(u, v, 1 / 2) \\
& \quad=4 u v-4 u \ln (1+v)-4 v \ln (1+u) \\
& \quad-4 u v\left[(1+u)^{-1}+(1+v)^{-1}-1\right]+2(u+v-1)^{+} /(u+v) .
\end{aligned}
$$

Thus, to complete the computation it remains only to determine $C(u, v, 1-)$. For this, we need the distribution of $K(x, 1-) K(y, 1-)$ which is deducible from the 
following in which we represent the two ordered spacings by $(1-U) / 2$ and $(1+U) / 2$ with $U$ being a $\operatorname{Unif}(0,1)$ r.v. For $0 \leq x \leq y \leq 1$,

$$
\begin{aligned}
P[K(x, 1-)=2, K(y, 1-)=2] & =P[(1+U) / 2 \leq x], \\
P[K(x, 1-)=1, K(y, 1-)=2] & =P[(1-U) / 2<x<(1+U) / 2<y] \\
& =P[(1-2 x) \vee(2 x-1)<U<2 y-1]
\end{aligned}
$$

and

$$
\begin{aligned}
P[K(x, 1-)=1, K(y, 1-)=1] & =P[(1-U) / 2<x \leq y<(1+U) / 2] \\
& =P[U>(1-2 x) \vee(2 y-1)] .
\end{aligned}
$$

Since $E K(x, 1-)=\mu(x, 1-)=2 x$, straightforward computations lead to

$$
C(u, v, 1-)=2 u-4 u v+2(u+v-1)^{+} .
$$

A combination of (7.2), (7.7) and (7.8) completes the proof of the following theorem.

THEOREM 7.1. For $0<u \leq v \leq 1$,

$$
\operatorname{Cov}(K(u s, s), K(v s, s))=C(u, v, s)=\frac{\alpha(u, v)}{s}, \quad 0<s \leq 1 / 2,
$$

where

$$
\begin{aligned}
\alpha(u, v)= & \frac{1}{2} C(u, v, 1 / 2) \\
= & -12 u v+4 u v\left\{(1+v)^{-1}+(1+u)^{-1}\right\}+4 u \ln (1+v) \\
& +4 v \ln (1+u)+2 u+2\left((u+v-1)^{+}\right)^{2}(u+v)^{-1}
\end{aligned}
$$

with $(x)^{+}=\max (0, x)$.

As a corollary of this result, the covariance of the process $W(\cdot, s)$, which is defined in (1.18) and has mean zero on $[0,1]$ by (1.8), is

$$
\operatorname{Cov}(W(u, s), W(v, s))=(s / 2) C(u, v, s)=\frac{1}{2} \alpha(u, v)
$$

for $0 \leq u \leq v \leq 1$ and $0<s \leq 1 / 2$. Note also that $\alpha(1,1) / 2=4 \ln 2-5 / 2=\sigma^{2}$; see Theorem 4.1. Clearly, the limiting process $W(\cdot)$ has the same covariance. By (1.19) this would mean that the covariance function for a limiting $V^{*}$-process for the normalized spacings would become

$$
\begin{aligned}
\operatorname{Cov}\left(V^{*}(u), V^{*}(v)\right) \\
=u(1-v)+(8 \ln 2-3) u v-2 u \ln (1+v)-2 v \ln (1+u) \\
\quad+\left\{(u+v-1)^{+}\right\}^{2} /(u+v)
\end{aligned}
$$


for $0 \leq u \leq v \leq 1$. More importantly, however, the covariances for the limiting $V$-process of Theorem 6.1 for the relative spacings becomes, by (7.10) and (1.20),

$$
\begin{aligned}
\operatorname{Cov}(V(u), V(v))= & u(1-v)-u v\left(\frac{3}{2}-\frac{1}{1+u}-\frac{1}{1+v}\right) \\
& +\left\{(u+v-1)^{+}\right\}^{2} /(u+v)
\end{aligned}
$$

for $0 \leq u \leq v \leq 1$. Observe that this latter covariance is zero at $V=1$ since $V$ is a tied-down process, whereas the untied process $V^{*}$ has variance at $v=1$ of $\sigma^{2}=4 \ln 2-5 / 2$; compare Theorem 3.1 and the difference between $V$ and $V^{*}$, which is seen through (1.19) and (1.20) to be $V(1)-V^{*}(1)=W(1)$.

REMARK. The focus of this paper has been solely upon the interval-splitting procedure of Kakutani (1975), and the methodologies required to obtain the weak convergence limits for the two main empirical processes under the particular dependence structure determined by this procedure. The paper therefore extends in a natural way the strong law or Glivenko-Cantelli results previously obtained for the Kakutani model; compare Lootgieter (1977), van Zwet (1978) and Pyke (1980).

Generalizations of the Kakutani procedure have been proposed. For example, the splitting random variables, $\left\{U_{i}\right\}$ in this paper, could have distributions other than uniform. Alternatively, procedures could allow for random selection of the interval to be split, rather than restricting it to be always the longest interval. Glivenko-Cantelli results for generalized procedures of these types have been studied in Brennan and Durrett (1987) and papers referenced therein. It is an open question whether weak convergence results for the analogous empirical processes can also be derived by the methodologies of this paper.

Other related references are Sibuya and Itoh (1987) and Komaki and Itoh (1992).

During the preparation of this paper, the authors had discussions with P. Diaconis and M. Shahshahani about their interests in this and related work. In particular, correspondence from P. Diaconis described calculations involving moments of the trace of a random $n \times n$ permutation matrix on the one hand, and of a random $n \times n$ orthogonal matrix on the other hand, for which the first $n$ (resp., $2 n+1)$ moments are exactly the moments of a Poisson (resp. normal) random variable. The connection with our work lies in the loose similarity with the type of result contained in our Theorem 2.2 in which an increasing number of moments become constant as a parameter, $1 / t$ increases.

\section{REFERENCES}

Anscombe, P. (1952). Large-sample theory of sequential estimation. Proc. Cambridge Philos. Soc. 45 600-607. 
BillingSLey, P. (1968). Convergence of Probability Measures. Wiley, New York.

Brennan, M. D. and Durrett, R. (1987). Splitting intervals. II. Limit laws for lengths. Probab. Theory Related Fields 75 109-127.

CSÖRGó, S. (1974). On weak convergence of the empirical process with random sample size. Acta Sci. Math. Szeged 36 17-25.

DONSKER, M. (1952). Justification and extension of Doob's heuristic approach to the KolmogorovSmirnov theorems. Ann. Math. Statist. 23 277-281.

Gihman, I. I. and SkOROHOD, A. V. (1974). The Theory of Stochastic Processes I. Springer, New York.

Kakutani, S. (1975). A problem of equidistribution on the unit interval [0,1]. Proceedings of Oberwolfach Conference on Measure Theory. Lecture Notes in Math. 541 369-376. Springer, Berlin.

Klatssen, C. A. J. and Wellner, J. A. (1992). Kac empirical processes and the bootstrap. In Proceedings of the Eighth International Conference on Probability in Banach Spaces (M. Hahn and J. Kuebs, eds.) 411-429. Birkhäuser, Boston.

KomaKi, F. and Iтон, Y. (1992). A unified model for Kakutani's interval splitting and Renyi's random packing. Adv. in Appl. Probab. 24 502-505.

Lootgieter, J. C. (1977). Sur la répartition des suites de Kakutani (I). Ann. Inst. H. Poincaré Ser. B $13385-410$.

PYKE, R. (1965). Spacings. J. Roy. Statist. Soc. Ser. B 27 395-449.

PYKE, R. (1968). The weak convergence of the empirical process with random sample size. Proc. Cambridge Philos. Soc. 64 155-160.

PYKE, R. (1980). The asymptotic behavior of spacings under Kakutani's model for interval subdivision. Ann. Probab. 8 157-163.

SibuYA, M. and ITOH, Y. (1987). Random sequential bisection and its associated binary tree. Ann. Inst. Statist. Math. 39 69-84.

VAN ZWET, W. R. (1978). A proof of Kakutani's conjecture on random subdivision of longest intervals. Ann. Probab. 6 133-137.

WEISS, L. (1955). The stochastic convergence of a function of sample successive differences. Ann. Math. Statist. 26 532-536.

DEPARTMENT OF MATHEMATICS

UNIVERSITY OF WASHINGTON

SEATTLE, WASHINGTON 98195

USA

E-MAIL:pyke@math.washington.edu
DEPARTMENT OF MATHEMATICS

UNIVERSITY OF LEIDEN

P.O. BOX 9512

2300 RA LEIDEN

THE NETHERLANDS

E-MAIL:vanzwet@math.leidenuniv.nl 